\title{
REVERSE TIME MIGRATION IN THE FREQUENCY DOMAIN BY THE RAPID EXPANSION METHOD
}

\author{
Protásio N. Andrade ${ }^{1,2}$, Reynam C. Pestana² and Daniel E. Revelo ${ }^{3}$
}

\begin{abstract}
This paper proposes and describes the implementation of a new depth migration method in the frequency domain. The method, based in the reverse time migration (RTM) technique, extrapolates wavefields from the source and receivers to obtain migrated seismic images that are built directly into the frequency domain. In the proposed method, wavefields are propagated in the time domain and are then transformed into the frequency domain at each time extrapolation step through the discrete Fourier transform. Neither the forward nor backward wavefield is needed to be stored in memory or read from disk storage. To speed up the migration algorithm, the discrete Fourier transform kernel for each frequency is computed and salved before the time extrapolation procedure. At the imaging condition phase, both source and receiver wavefields are at the same frequency, so that, the construction of the image occurs by multiplying the forward source propagated wavefield with the backward propagated of the receivers wavefield for each frequency component. Subsequently, saving the source field at each step to later correlate it with the back propagated receiver wavefield, usually done in conventional RTM, becomes unnecessary. Nor is it necessary to invert a matrix for each frequency component, which is done in the migration technique that uses the Helmholtz equation solution in the frequency domain. Thus, the migration procedure in the frequency domain being proposed is more efficient from a computational point of view, and can also produce high quality migrated images as those produced by conventional RTM. The rapid expansion method (REM) is used for seismic forward modeling, which extrapolated data with good precision and free of numerical dispersion. Thus, with the transformed data at each step in the frequency domain, it is possible to construct high quality, in-depth seismic images at a lower computational cost. Moreover, this frequency domain migration with REM is an atractive strategy to design robust inverse algorithms, especially for 3D problems. To demonstrate the efficiency and applicability of the proposed method, two synthetic models were used and their results showed high quality images equivalent to those obtained by conventional RTM and thus proving the vality of the method.
\end{abstract}

Keywords: wave equation migration, depth migration, imaging condition, frequency domain migration.

RESUMO. Um método de migração em profundidade no domínio da frequência é proposto e implementado. 0 método consiste na extrapolação dos campos de ondas da fonte e dos receptores e baseia-se na técnica de migração reversa no tempo (da sigla em inglês, RTM), obtendo imagens sísmicas migradas, construídas diretamente no domínio da frequência. No método que estamos propondo, os campos de ondas são propagados no domínio do tempo e a cada passo de extrapolação são transformados para o domínio da frequência, através da transformada de Fourier discreta (do inglês, on-the-fly transform). Para acelerar 0 algoritmo de migração, 0 kernel da transformada de Fourier é calculado fora do loop do tempo. Além disso, na etapa de condição da imagem, os campos de onda, tanto da fonte como dos receptores, são calculados no mesmo instante de tempo, ou seja, a construção da imagem se dá através da multiplicação do campo de onda da fonte com o campo retropropagado dos receptores, para cada componente de frequência. Portanto, não precisamos salvar o campo da fonte a cada passo no tempo para posteriormente correlacionar com o campo de onda retropropagado dos receptores, como é usualmente feito na RTM convencional, nem é preciso inverter uma matriz para cada componente de frequência, como é realizado normalmente pela técnica de migração no domínio da frequência, utilizando a solução da equação de Helmholtz. Desta forma, o procedimento de migração no domínio da frequência que estamos propondo se torna mais eficiente do ponto de vista computacional, podendo produzir imagens migradas de alta qualidade, quando comparadas às obtidas através da RTM convencional no domínio do tempo. Para a extrapolação dos campos de ondas no tempo foi empregado 0 método de expansão rápida (da sigla em inglês, REM), que permite a extrapolação dos dados com boa precisão e livres de dispersão numérica. Desta forma, com os dados transformados para o domínio da frequência, a cada passo no tempo, é possível a construção de imagens sísmicas em profundidade de boa qualidade e a um menor custo computacional. Para demonstrar a eficiência e aplicabilidade do método proposto, dois modelos sintéticos foram usados e seus resultados apresentaram imagens de alta qualidade equivalentes às obtidas pela RTM convencional.

Palavras-chave: equação de migração da onda, migração, condição de imagem, migração no domínio da frequência.

\footnotetext{
1 Universidade Salvador (UNIFACS), Architecture, Engineering and Information Technology School (AEITS), Laureate International Universities, Rua Doutor José Peroba, 41770-235 Salvador, BA, Brazil. Phone: +55(71) 3021-2800/99206-5486 - E-mail: protasio.andrade@unifacs.br

2 Universidade Federal da Bahia (UFBA), Center for Research in Geophysics and Geology (CPGG), Rua Barão de Geremoabo, 40170-115 Salvador, BA, Brazil. Phone: +55(71)3283-8551 - E-mails: protasio.nery@ufba.br; reynam@cpgg.ufba.br

3 Universidade Federal da Bahia (UFBA), Center for Research in Geophysics and Geology (CPGG), Rua Barão de Geremoabo, 40170-115 Salvador, BA, Brazil. Phone: +55(71)3283-8551/99112-8368 - E-mail: dapraez@ufba.br
} 


\section{INTRODUCTION}

Reverse time migration (RTM) using the two-way acoustic wave equation is not a new concept. It was introduced in the late 1970's by Hemon (1978), and has the advantage of handling multipath and steeply-dipping reflectors (Baysal et al., 1983; Whitmore, 1983; McMechan, 1983; Loewenthal \& Mufti, 1983). However, at the beginning this method was not commonly used in the seismic exploration industry because it required a great amount of computer memory and computing time. Instead, the Kirchhoff integral method and the one-way wave equation (OWE) methods were widely used because of the low computational cost and lower memory demand. With the rapid growth of computer technology, 3D prestack RTM is being used to address the imaging challenges posed by sub salt and other complex subsurface targets.

By using the full wave equation, RTM implicitly includes multiple arrival paths and has no dip limitation, thus enabling the imaging of complex reflectors. For post-stack seismic data, RTM is performed by propagating the recorded wavefield backward in time into the subsurface with half the velocity of the medium. At time $t=0$, the back propagated wavefield provides the subsurface image. For prestack data, the RTM uses a time-coincidence imaging condition (Claerbout, 1971). Typically in prestack RTM, each shot is forward propagated and the recorded data is back propagated. The migrated section is obtained by applying the imaging condition, where the source and receiver wavefields are cross-correlated, with zero-lag, managing to correctly position the reflectors in depth and collapse diffractions. RTM can also be considered the inverse operation of forward modeling, where the same numerical modeling code used for forward modeling can be used for RTM.

RTM is currently computationally feasible even in $3 D$ prospects, but still requires a high computational demand. A common RTM implementation, for example, uses small steps in time to avoid numerical instability and low frequency content in order to reduce numerical dispersion in the migrated data. This is implied in the resampling of data and primarily in the filtering to remove high frequency components in 3D dataset, as the RTM algorithm in large seismic surveys has a high computational demand that requires unacceptably long run times, even with a computer cluster. Recently however, many new algorithms have been developed to overcome this problem related with the time step.

Soubaras \& Zhang (2008) introduced a two-step marching method that permits data extrapolation with a larger time step. Zhang \& Zhang (2009) proposed a one-step extrapolation method that is implemented on the basis of the optimized separable approximation (OSA) (Song, 2008). Pestana \& Stoffa (2009) intro- duced RTM using recursive time stepping based on the rapid expansion method (REM) (Tal-Ezer et al., 1987). Thus, it is possible to perform migration with longer time steps in a stable manner and without numerical noise caused by numerical dispersion.

Migration of seismic data is commonly carried out in the time domain. The classic RTM algorithms in the time domain are known to be computationaly $1 / 0$ intensive, because the forward and back propagation wavefields have to be computed and stored. If the correlation between these fields is carried out during the time-reversed computation of the receiver data, only snapshots of the forward source wavefield have to be stored. To reduce the overhead of storing snapshots, severals strategies have been proposed and applied with success.

Another alternative is to perform seismic migration in the frequency domain, where the most common form results from the solution of the Helmholtz equation. In the simplified matrix form for direct modeling, we have: $S p=f$, where: $p$ is the pressure field, $f$ is the source, and $S$ is the direct modeling operator. The inverted matrix, $S^{-1}$, for example, can be applied to each source, $p=S^{-1} f$, providing the source wavefield for each frequency component (Shabelansky, 2007). Thus, the forward and backward propagation are computed and there is no need to store the wavefields in disk.

Migration in the frequency domain requires the selection of a set of frequencies that avoids spatial aliasing. The seismic data and the source signature are transformated into frequency domain. For each frequency the Helmholtz is solved. An important advantage of migration in the frequency domain is that the cross-correlation needed for imaging condition becomes a simples multiplication of the wavefields at each frequency, followed by a summation over selected frequencies.

One of the problems with the migration in the frequency domain is the necessity to solve a sparse indefinite linear system of equations, which arise from the discretization of the Helmholtz equation, whereas in the time domain, the discretization of the wave equation in space and time leads to an explicit time marching scheme.

Methods that make the matrix less sparse together with efficient computational software such as MUMPS (Multifrontal Massively Parallel Sparse Direct Solver) have been used to solve this problem. Furthermore, the RTM in the frequency domain with a direct solver executes much more efficient for multiple-shot than time domain RTM does for a 2D case. However, for the 3D application of frequency domain RTM, the direct method decomposition has a weakness of taking dramatic memory an calculation amount because aside from inverting the matrix for each 
frequency, matrices $L$ and $U$ must be stored for each frequency in order to calculate the wavefield (Operto et al., 2007) and makes it difficult to run on full scale problem efficiently. Alternatively, the iterative solvers are employed to lessen the memory the occupation, but this will considerably weaken the advantage of frequency domain RTM because multi-shot modeling cannot be performed simultaneously with one decomposed matrix just as the direct solver did. The iterative solutions do not consume a great amount of memory, however they do suffer from high frequencies, showing poor convergence rates. An alternative to this problem is the multi-scale approach, which attempts to solve the problem by dividing the data into frequency bands, improving the convergence.

Despite the many approaches in addressing the 3D problem in frequency domain, it remains a great challenge due to its high computational demand (Operto et al., 2007). New alternatives have been proposed to solve the $3 \mathrm{D}$ problem, one of which is transforming time domain data into frequency. This transformation is generally implemented during propagation in time by applying the discrete Fourier transform (DFT) for each wavefield snapshot, also known as on-the-fly transform (Furse, 2000; Nihei \& Li, 2007; Sirgue et al., 2008). More recently, Chu \& Stoffa (2012) used this technique by applying the REM method in its non-recursive form, then applying the discrete Fourier transform during data modeling.

Usually in the time domain, the most common methods for seismic forward modeling are finite-difference or pseudospectral methods. In our work we use the REM (Pestana \& Stoffa, 2010) method because it is numerically stable and free of numerical dispersion, generating better wavefield propagation results (Tessmer, 2011). The REM method developed by Pestana \& Stoffa (2010) calculates the wave propagation for each step in time, whereas the method Chu \& Stoffa (2012) use computes the wavefield through non-recursive REM. In the method proposed for this study, wavefield propagation is done through the nonrecursive REM in time, and a discrete Fourier transform is done at each time step.

The imaging condition in the frequency domain is generated by taking the real value of the source wavefield multiplied by the receiver wavefields. In our implementation, we do not need to save the source wavefield for all instances of time and correlate it with the receivers wavefield during back propagation. As we move through time, the direct propagation of the source wavefield and the back propagation of the receiver wavefield are performed inside the same loop in time. Moreover, the Fourier transform kernel is calculated outside the time loop, thus allowing a greater computational efficiency for the algorithm.

In the following theory section, we present the development relative to the imaging condition based on the scattering theory with Born approximation, as well as the procedure used to calculate the wavefields in the frequency domain where the discrete Fourier transform was applied at each extrapolation step in time. Finally, we show some numerical examples and compare the computational performance on a $2 \mathrm{D}$ example of our proposed frequency domain RTM with the convention time domain RTM using checkpointing strategy that only save the source wavefield at predefined checkpoints in time and recomputing the wavefields at other instances from theses checkpoints.

\section{THEORY}

The conventional migration approach proposed by Claerbout (1971) provides a subsurface image by applying the adjoint modeling operator to the seismic data. This procedure can be considered the first iteration of the full waveform inversion method (FWI) (Ren et al., 2013).

To determine the migration operator, we start from the direct propagation representation from the complete acoustic wave in the frequency domain via the Helmholtz equation solution, then employ the Born approximation.

Helmholtz equation can be written as follows:

$$
\left[\nabla^{2}+\omega^{2} \mathbf{m}^{2}(\mathbf{x})\right] \mathbf{P}\left(\mathbf{x}, \mathbf{x}_{\mathbf{s}}, \omega\right)=\mathbf{F}(\mathbf{x}, \omega),
$$

where

$$
\nabla^{2}=\left(\frac{\partial^{2}}{\partial x^{2}}+\frac{\partial^{2}}{\partial y^{2}}+\frac{\partial^{2}}{\partial z^{2}}\right),
$$

$\omega$ is the angular frequency, $\mathbf{x}=(x, y, z)$ the vector position and the parameter model is the slowness, defined as: $\mathbf{m}(\mathbf{x})=$ $1 / v(\mathbf{x}) . \quad \mathbf{P}$ is the pressure field, $\mathbf{x}_{\mathbf{s}}$ is the source position and $\mathbf{x}$ is the listening location. The source term is given by $\mathbf{F}(\mathbf{x}, \omega)=-\delta\left(\mathbf{x}-\mathbf{x}_{\mathbf{s}}\right) A(\omega)$, where $A(\omega)$ is the source spectrum.

We can represent the slowness model as being composed of a background model plus a disturbance, that is, $\mathbf{m}(\mathbf{x})=$ $\mathbf{m}_{o}(\mathbf{x})+\delta \mathbf{m}(\mathbf{x})$ and, consequently, the wavefield in the same way, $\mathbf{P}=\mathbf{P}_{o}+\delta \mathbf{P}$, where $\mathbf{m}_{o}$ is the background model and $\mathbf{P}_{o}$ is the propagated field in background model, $\delta \mathbf{m}$ is the perturbation and $\delta \mathbf{P}$ and scattered wavefield.

Our goal is to get an expression for $\delta \mathbf{P}$, which can be used to simulate the observed data $\left(\mathbf{d}_{o b s}\right)$. Figure 1 exemplifies the propagation of the source wavefield and the scattered wavefield, recorded along the receptors due to a disturbance in the slowness model. 


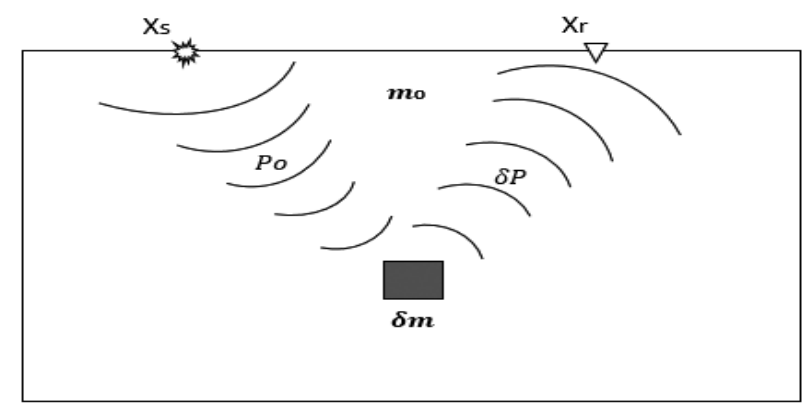

Figure 1 - Seismic wave scattering scheme: The source wavefield $\mathbf{P}_{o}$ propagates at constant background velocity, $c_{o}=1 / \mathbf{m}_{o}$, until it interacts with a scatterer (slowness perturbation, $\delta \mathbf{m}$ ) which acts as a secondary source and scatterers the incoming wavefield. The scattered wavefield $\delta \mathbf{P}$ propagates at the background $c_{0}$ and is recorded by receivers $\mathbf{x}_{\mathbf{r}}$.

In order to obtain a solution to Eq. (1), we can write that:

$$
\mathbf{P}\left(\mathbf{x}, \mathbf{x}_{\mathbf{s}}, \omega\right)=A(\omega) \mathbf{G}\left(\mathbf{x}, \mathbf{x}_{\mathbf{s}}, \omega\right)
$$

where $\mathbf{G}\left(\mathbf{x}, \mathbf{x}_{\mathbf{s}}, \omega\right)$ is the Green's function that satisfies the following Helmholtz equation:

$$
\left[\nabla^{2}+\omega^{2} \mathbf{m}^{2}(\mathbf{x})\right] \mathbf{G}\left(\mathbf{x}, \mathbf{x}_{\mathbf{s}}, \omega\right)=-\delta\left(\mathbf{x}-\mathbf{x}_{\mathbf{s}}\right) .
$$

For the background field we have that:

$$
\mathbf{P}_{o}\left(\mathbf{x}, \mathbf{x}_{\mathbf{s}}, \omega\right)=A(\omega) \mathbf{G}_{o}\left(\mathbf{x}, \mathbf{x}_{\mathbf{s}}, \omega\right),
$$

where $\mathbf{G}_{o}\left(\mathbf{x}, \mathbf{x}_{\mathbf{s}}, \omega\right)$ is the Green's function for the background model that satisfies the following equation:

$$
\left[\nabla^{2}+\omega^{2} \mathbf{m}_{o}^{2}(\mathbf{x})\right] \mathbf{G}_{o}\left(\mathbf{x}, \mathbf{x}_{\mathbf{s}}, \omega\right)=-\delta\left(\mathbf{x}-\mathbf{x}_{\mathbf{s}}\right)
$$

Substituting $\mathbf{m}^{2}(\mathbf{x})$ by $\left(\mathbf{m}_{o}(\mathbf{x})+\delta \mathbf{m}(\mathbf{x})\right)^{2}$ in Eq. (1), expanding the squared term and neglecting the term $\delta \mathbf{m}^{2}$, we have:

$$
\left[\nabla^{2}+\omega^{2} \mathbf{m}_{o}^{2}(\mathbf{x})\right] \mathbf{P}\left(\mathbf{x}, \mathbf{x}_{\mathbf{s}}, \omega\right)=\mathbf{F}(\mathbf{x}, \omega)-2 \omega^{2} \mathbf{m}_{o}(\mathbf{x}) \delta \mathbf{m}(\mathbf{x}) \mathbf{P}\left(\mathbf{x}, \mathbf{x}_{\mathbf{s}}, \omega\right) .
$$

According to Green's theorem, multiplying both sides of the Eq. (6) by $\mathbf{G}_{o}\left(\mathbf{x}, \mathbf{x}^{\prime}, \omega\right)$ and integrating over the whole volume containing the $\mathbf{x}^{\prime}$ index, gives the the Lippmann-Schwinger equation, which is an integral equation with unknown $\mathbf{P}\left(\mathbf{x}, \mathbf{x}_{\mathbf{s}}, \omega\right)$ on both sides and $\mathbf{r}\left(\mathbf{x}^{\prime}\right)=2 \mathbf{m}_{o}\left(\mathbf{x}^{\prime}\right) \delta \mathbf{m}\left(\mathbf{x}^{\prime}\right)$ representing the reflectivity model. Applying the Born's approximation, that is, $\mathbf{G}\left(\mathbf{x}^{\prime}, \mathbf{x}_{\mathbf{s}}, \omega\right)=$ $\mathbf{G}_{o}\left(\mathbf{x}^{\prime}, \mathbf{x}_{\mathbf{s}}, \omega\right)$, we obtain the scattered wavefield that is given as:

$$
\mathbf{P}\left(\mathbf{x}, \mathbf{x}_{\mathbf{s}}, \omega\right)-\mathbf{P}_{o}\left(\mathbf{x}, \mathbf{x}_{\mathbf{s}}, \omega\right)=\omega^{2} \int \mathbf{r}\left(\mathbf{x}^{\prime}\right) A(\omega) \mathbf{G}_{o}\left(\mathbf{x}^{\prime}, \mathbf{x}_{\mathbf{s}}, \omega\right) \mathbf{G}_{o}\left(\mathbf{x}, \mathbf{x}^{\prime}, \omega\right) d \mathbf{x}^{\prime}
$$

In Eq. (7), evaluating the field along the receivers, that is, $\mathbf{x}=\mathbf{x}_{\mathbf{r}}$, and having $\delta \mathbf{P}=\mathbf{P}-\mathbf{P}_{o}$ we get:

$$
\delta \mathbf{P}\left(\mathbf{x}_{\mathbf{r}}, \mathbf{x}_{\mathbf{s}}, \omega\right)=\omega^{2} \int \mathbf{r}\left(\mathbf{x}^{\prime}\right) A(\omega) \mathbf{G}_{o}\left(\mathbf{x}^{\prime}, \mathbf{x}_{\mathbf{s}}, \omega\right) \mathbf{G}_{o}\left(\mathbf{x}_{\mathbf{r}} \cdot \mathbf{x}^{\prime}, \omega\right) d \mathbf{x}^{\prime} .
$$

Aside from this, Eq. (8), which represents the direct problem, can be rewritten in its linearized form through the following relation:

$$
\delta \mathbf{P}\left(\mathbf{x}_{\mathbf{r}}, \mathbf{x}_{\mathbf{s}}, \omega\right)=\mathbf{L}\left(\mathbf{x}, \mathbf{x}_{\mathbf{r}}, \mathbf{x}_{\mathbf{s}}, \omega\right) \mathbf{r}(\mathbf{x}) .
$$


Thus the forward modeling operator is explicitly given by:

$$
\mathbf{L}\left(\mathbf{x}, \mathbf{x}_{\mathbf{r}}, \mathbf{x}_{\mathbf{s}}, \omega\right)=\omega^{2} A(\omega) \mathbf{G}_{o}\left(\mathbf{x}, \mathbf{x}_{\mathbf{s}}, \omega\right) \mathbf{G}_{o}\left(\mathbf{x}_{\mathbf{r}}, \mathbf{x}, \omega\right)
$$

where $\mathbf{G}_{o}\left(\mathbf{x}, \mathbf{x}_{\mathbf{s}}, \omega\right)$ is the Green's function of the source at position $\mathbf{x}_{\mathbf{s}}$ to the scattering position $\mathbf{x}$, and $\mathbf{G}_{o}\left(\mathbf{x}_{\mathbf{r}}, \mathbf{x}, \omega\right)$ is the Green's function in position $\mathbf{x}$ to receiver $\mathbf{x}_{\mathbf{r}}$.

Therefore, by applying the adjoint of $\mathbf{L}$ to the observed data, $\delta \mathbf{P}=d_{o b s}$, and taking into account the reciprocity relation of the Green's function, $\mathbf{G}_{o}\left(\mathbf{x}_{\mathbf{r}}, \mathbf{x}, \omega\right)=\mathbf{G}_{o}\left(\mathbf{x}, \mathbf{x}_{\mathbf{r}}, \omega\right)$, we have:

$$
\mathbf{r}(\mathbf{x})=\mathbf{L}^{\dagger} \delta \mathbf{P}
$$

which can be rewritten as:

$$
\mathbf{r}(\mathbf{x})=\operatorname{Re}(\sum_{\omega} \omega^{2} \sum_{\mathbf{x}_{\mathbf{s}}} \sum_{\mathbf{x}_{\mathbf{r}}} \overbrace{A(\omega)^{\dagger} \mathbf{G}_{o}^{\dagger}\left(\mathbf{x}, \mathbf{x}_{\mathbf{s}}, \omega\right)}^{\text {forward propagated wavefield }} \overbrace{\mathbf{G}_{o}^{\dagger}\left(\mathbf{x}, \mathbf{x}_{\mathbf{r}}, \omega\right) \delta \mathbf{P}\left(\mathbf{x}_{\mathbf{s}}, \mathbf{x}_{\mathbf{r}}, \omega\right)}^{\text {back propagated wavefield }}),
$$

where $\dagger$ represents the transposed conjugation.

The Eq. (12) expresses the imaging condition for frequency migration, where the image is constructed from the sum of all frequencies and sources, which is attained by multiplying the directly propagated source field with the observed data field back propagated to the same frequency.

The above Eq. (12) is the same one presented in the works of Pan (2006) and Maaref et al. (2008) which also uses Born approximation, as well as in the works of Dai et al. (2012); Ren et al. (2013).

Although the deduction presented above requires the Green's function $\mathbf{G}_{o}^{\dagger}\left(\mathbf{x}, \mathbf{x}_{\mathbf{r}}, \omega\right)$ to be calculated for each receiver independently, we will show below that these Green's functions can be calculated simultaneously for all receptors (Hammad, 2010), as the sum of these Green's functions is valid because of the linearity of the wave equation.

To simplify the notation, we remove the dependence of the source position $\mathbf{x}_{\mathbf{s}}$ from the observed data $\delta \mathbf{P}\left(\mathbf{x}_{\mathbf{r}}, \mathbf{x}_{\mathbf{s}}, \omega\right)$, in Eq. (12), and fix for the frequency and the shot. We start by showing the calculations for two receivers and then extend to other receivers. After these simplifications, Eq. (12) can be rewritten as:

$$
\mathbf{r}(\mathbf{x})=\omega^{2} A(\omega) \mathbf{G}_{o}^{\dagger}\left(\mathbf{x}, \mathbf{x}_{\mathbf{s}}, \omega\right) \delta \mathbf{P}\left(\mathbf{x}_{\mathbf{r} 1}, \omega\right) \mathbf{G}_{o}^{\dagger}\left(\mathbf{x}, \mathbf{x}_{\mathbf{r} 1}, \omega\right)+\omega^{2} A(\omega) \mathbf{G}_{o}^{\dagger}\left(\mathbf{x}, \mathbf{x}_{\mathbf{s}}, \omega\right) \delta \mathbf{P}\left(\mathbf{x}_{\mathbf{r} 2}, \omega\right) \mathbf{G}_{o}^{\dagger}\left(\mathbf{x}, \mathbf{x}_{\mathbf{r} 2}, \omega\right)
$$

Now, using the following Green's function property, we can also write that:

$$
\mathbf{P}\left(\mathbf{x}, \mathbf{x}_{\mathbf{r} 1}, \omega\right)=\delta \mathbf{P}\left(\mathbf{x}_{\mathbf{r} 1}, \omega\right) \mathbf{G}_{o}^{\dagger}\left(\mathbf{x}, \mathbf{x}_{\mathbf{r} 1}, \omega\right) .
$$

Based on the Eq. (2), we have that $\delta \mathbf{P}\left(\mathbf{x}_{\mathbf{r} 1}, \omega\right)$ exercises the same role as the source term, $A(\omega)$. Now, for the second receiver we have:

$$
\mathbf{P}\left(\mathbf{x}, \mathbf{x}_{\mathbf{r} 2}, \omega\right)=\delta \mathbf{P}\left(\mathbf{x}_{\mathbf{r} 2}, \omega\right) \mathbf{G}_{o}^{\dagger}\left(\mathbf{x}, \mathbf{x}_{\mathbf{r} 2}, \omega\right) .
$$

Considering Eqs. (14) and (15), Eq. (13) can be rewritten as:

$$
\mathbf{r}(\mathbf{x}, \omega)=\omega^{2} A(\omega) \mathbf{G}_{o}\left(\mathbf{x}, \mathbf{x}_{\mathbf{s}}, \omega\right)\left[\mathbf{P}\left(\mathbf{x}, \mathbf{x}_{\mathbf{r} 1}, \omega\right)+\mathbf{P}\left(\mathbf{x}, \mathbf{x}_{\mathbf{r} 2}, \omega\right)\right] .
$$

Introducing wavefield $\tilde{\mathbf{P}}\left(\mathbf{x}, \mathbf{x}_{\mathbf{r} 1}, \mathbf{x}_{\mathbf{r} 2}, \omega\right)$, which is obtained by adding the fields defined in Eqs. (14) and (15).

$$
\tilde{\mathbf{P}}\left(\mathbf{x}, \mathbf{x}_{\mathbf{r} 1}, \mathbf{x}_{\mathbf{r} 2}, \omega\right)=\left[\mathbf{P}\left(\mathbf{x}, \mathbf{x}_{\mathbf{r} 1}, \omega\right)+\mathbf{P}\left(\mathbf{x}, \mathbf{x}_{\mathbf{r} 2}, \omega\right)\right],
$$


and finally extending to all other receivers, we obtain the following wavefield:

$$
\tilde{\mathbf{P}}\left(\mathbf{x}, \mathbf{x}_{\mathbf{r} 1}, \mathbf{x}_{\mathbf{r} 2}, \ldots, \mathbf{x}_{\mathbf{r n}}, \omega\right)=\left[\mathbf{P}\left(\mathbf{x}, \mathbf{x}_{\mathbf{r} 1}, \omega\right)+\mathbf{P}\left(\mathbf{x}, \mathbf{x}_{\mathbf{r} 2}, \omega\right)+\cdots+\mathbf{P}\left(\mathbf{x}, \mathbf{x}_{\mathbf{r n}}, \omega\right)\right] .
$$

In this way, the wavefield $\tilde{\mathbf{P}}$, defined in the Eq. (18), represents the back propagated wavefield of all receivers and Eq. (13) is rewritten as follows:

$$
\mathbf{r}(\mathbf{x}, \omega)=\omega^{2} A(\omega) \mathbf{G}_{o}\left(\mathbf{x}, \mathbf{x}_{\mathbf{s}}, \omega\right) \tilde{\mathbf{P}}\left(\mathbf{x}, \mathbf{x}_{\mathbf{r} 1}, \mathbf{x}_{\mathbf{r} 2}, \ldots, \mathbf{x}_{\mathbf{r n}}, \omega\right)
$$

In order to show that the receiver wavefields can be back propagated just one time, we can rewrite the Helmholtz equation as follows:

$$
\mathcal{L} \mathbf{P}\left(\mathbf{x}, \mathbf{x}_{\mathbf{r} 1}, \omega\right)=-A(\omega) \delta\left(\mathbf{x}-\mathbf{x}_{\mathbf{r} 1}\right)
$$

where

$$
\mathcal{L}=\left[\nabla^{2}+\omega^{2} \mathbf{m}^{2}(\mathbf{x})\right],
$$

represents the impedance matrix of the system.

Thus, the scattered and recorded wavefield at receiver $\mathbf{x}_{\mathbf{r} 1}$ satisfies the following equation:

$$
\mathcal{L} \mathbf{P}\left(\mathbf{x}, \mathbf{x}_{\mathbf{r} \mathbf{1}}, \omega\right)=-\delta \mathbf{P}\left(\mathbf{x}_{\mathbf{r} \mathbf{1}}, \omega\right) \delta\left(\mathbf{x}-\mathbf{x}_{\mathbf{r} \mathbf{1}}\right) .
$$

Likewise, for the receiver $\mathbf{x}_{\mathbf{r} 2}$, we also have:

$$
\mathcal{L} \mathbf{P}\left(\mathbf{x}, \mathbf{x}_{\mathbf{r} 2}, \omega\right)=-\delta \mathbf{P}\left(\mathbf{x}_{\mathbf{r} 2}, \omega\right) \delta\left(\mathbf{x}-\mathbf{x}_{\mathbf{r} 2}\right) .
$$

Adding Eq. (23) and Eq. (22) results in:

$$
\begin{gathered}
\mathcal{L}\left[\mathbf{P}\left(\mathbf{x}, \mathbf{x}_{\mathbf{r} 1}, \omega\right)+\mathbf{P}\left(\mathbf{x}, \mathbf{x}_{\mathbf{r} 2}, \omega\right)\right]= \\
-\delta \mathbf{P}\left(\mathbf{x}_{\mathbf{r} 1}, \omega\right) \delta\left(\mathbf{x}-\mathbf{x}_{\mathbf{r} \mathbf{1}}\right) \\
-\delta \mathbf{P}\left(\mathbf{x}_{\mathbf{r} 2}, \omega\right) \delta\left(\mathbf{x}-\mathbf{x}_{\mathbf{r} \mathbf{2}}\right)
\end{gathered}
$$

Now, considering the Eq. (22) for each receiver, taking the sum of all receivers, then substituting wavefield $\tilde{\mathbf{P}}$, defined in the Eq. (18), we obtain the following result:

$$
\begin{gathered}
\mathcal{L} \tilde{\mathbf{P}}\left(\mathbf{x}, \mathbf{x}_{\mathbf{r} 1}, \mathbf{x}_{\mathbf{r} 2}, \ldots, \mathbf{x}_{\mathbf{r n}}, \omega\right)= \\
-\delta \mathbf{P}\left(\mathbf{x}_{\mathbf{r} 1}, \omega\right) \delta\left(\mathbf{x}-\mathbf{x}_{\mathbf{r} 1}\right) \\
-\delta \mathbf{P}\left(\mathbf{x}_{\mathbf{r} 2}, \omega\right) \delta\left(\mathbf{x}-\mathbf{x}_{\mathbf{r} 2}\right) \\
-\ldots \delta \mathbf{P}\left(\mathbf{x}_{\mathbf{r n}}, \omega\right) \delta\left(\mathbf{x}-\mathbf{x}_{\mathbf{r n}}\right) .
\end{gathered}
$$

Therefore, Eq. (25) demonstrates that the wavefield at all receivers can be simultaneously back propagated, injecting all receivers recorded data as sources. In other words, it is not necessary to back propagate each receiver wavefield separately, reducing the back propagation of all receivers at each shot to one simple back propagation where all receivers are contemplated at once.

\section{Wavefield extrapolation by REM}

To calculate the receiver and source wavefields, we use REM proposed by Pestana \& Stoffa (2010), which is implemented through Eq. (26).

In this method, the wavefield is recursively extrapolated in time, unlike the original REM in its non-recursive form, initially proposed by Tal-Ezer et al. (1987) and modified by Kosloff et al. (1989). Using this approach, the extrapolation in time can be made within longer time intervals in a stable manner with better accuracy, and without presenting a numerical dispersion when compared to finite-difference and pseudo-spectral methods.

The solution of the wave equation, according to Pestana \& Stoffa (2010), using the REM is given by:

$$
\begin{gathered}
\mathbf{P}(t+\Delta t)+\mathbf{P}(t-\Delta t)= \\
2 \sum_{k=0}^{M} C_{2 k} J_{2 k}(R \Delta t) Q_{2 k}\left(\frac{i L}{R}\right) \mathbf{P}(t),
\end{gathered}
$$

where $J_{2 k}$ are the Bessel's functions and $Q_{2 k}\left(\frac{i L}{R}\right)$ are the modified Chebyshev polynomials. The value of $R$, used to normalize the polynomials of Chebyshev, is given by Tal-Ezer et al. (1987):

$$
R=\pi c_{\max } \sqrt{\left(\frac{1}{\Delta x}\right)^{2}+\left(\frac{1}{\Delta y}\right)^{2}+\left(\frac{1}{\Delta z}\right)^{2}},
$$

where $c_{\max }$ is the maximum velocity, $\Delta x, \Delta y$ and $\Delta z$ are the spatial samplings, based on velocity model discretization.

For REM, according to Tal-Ezer et al. (1987), the Eq. (26) converges exponentially. And for each extrapolation step in time $\Delta t$, the maximum number of terms $M$ is given by $M>\Delta t R / 2$ (Pestana \& Stoffa, 2010).

The change from time domain to frequency domain occurs throughout each extrapolation step in time, through the discrete Fourier transform, as suggested in the works of Furse (2000); Nihei \& Li (2007); Sirgue et al. (2008). 
The wavefield at each step in time, starting from Eq. (26), is transformed to the frequency domain through the following discrete Fourier transform:

$$
\mathbf{P}(\mathbf{x}, \omega)=\sum_{n=1}^{n t} e^{i \omega n \Delta t} \mathbf{P}(\mathbf{x}, n \Delta t),
$$

where $\Delta t$ is the time extrapolation interval, and $n t$ is the number of time intervals given by $n t=t_{\max } / \Delta t$.

To perform the reverse migration in time in its conventional form, we have to model the source field and save its snapshots. The data recorded along the receivers are then back propagated. As the field is back propagated at every step in time, the imaging condition, which is zero-lag cross-correlation of the source and receiver wavefields, is applied, thus obtaining the migrated seismic section.

RTM in its conventional form, as described above, must save the modeled source fields, that is, each snapshot of the propagation. In 3D cases, or even 2D for large models, extensive computational time will be required to read and write data to disk, making it problematic for RTM implementation. In some cases, this problem can be partially solved through the checkpoint technique, where some snapshots of the source propagation are saved, not necessarily all snapshots, and as the data is back propagated, the source field is reconstituted from the recorded snapshots.

In the proposed method, the kernel calculation of the discrete Fourier transform is done outside the time extrapolation loop, saving these kernels for each frequency component and time interval, then applying them to both source and receiver wavefields at each time step and frequency, thus generating their respective wavefields in frequency domain.

The imaging condition is applied after transforming the source wavefield (direct propagation) and the receivers field (reverse propagation) to the frequency domain using the Fourier transform kernel and multiplying these fields at each frequency. Thus, the migration of each common shot section is performed and at the end of the extrapolation process of all shots the final migrated image is generated from the sum of the partial images of each common shot section.

Algorithm 1 shows the pseudo-code for the RTM procedure described above. In our implementation, the forward and backward propagations are computed in parallel for each shot an there is no need to store the wavefields on disk. Basically, for each time step, source and receivers wavefield are forward and backward propagated by REM and for each frequency are then multiplied with each other. Only two wavefields are kept in memory, whereas in time, all consecutive wavefields for the forward propagation need to be stored. The flowchart the proposed RTM in the frequency domain algorithm is presented in Figure 2.

\section{RESULT}

To verify the efficiency of the proposed algorithm, two synthetic models were used to test it. First, a simple model with four-layers with velocities of $2000 \mathrm{~m} / \mathrm{s}, 3000 \mathrm{~m} / \mathrm{s}, 4000 \mathrm{~m} / \mathrm{s}$ and $2000 \mathrm{~m} / \mathrm{s}$, respectively, is shown in Figure 3. The spatial sampling is $10 \mathrm{~m}$ in both directions and the model is discretized and represented by a $315 \times 195$ mesh. A Ricker pulse was used to model the data, with a peak frequency of $25 \mathrm{~Hz}$, producing 62 shots with each shot having 315 traces and each trace having 1500 samples with a time sampling interval of $1 \mathrm{~ms}$.

To test the proposed method, in Figure 4 we show a snapshot obtained from the modeling of a source positioned in the center of the model, using the velocity model shown in Figure 3. Figure 4(a) shows the wavefield propagated in the time domain, snapshot at $0.84 \mathrm{~s}$, and Figures $4(\mathrm{~b}-\mathrm{f})$ show the real part of the wavefield (snapshot at same time) obtained by a summation over the selected frequency bands of $0-10 \mathrm{~Hz}, 0-30 \mathrm{~Hz}, 0-60 \mathrm{~Hz}$, 0-90 Hz and 0-120 Hz, respectively, computed using the proposed method.

From these results we can verify that as the frequency band is increased, and all the corresponding wavefields at each frequency are summed together, we obtain a result equivalent to that obtained from time directly (Fig. 4(a)). Therefore, using this simples experiment, we have demonstrated that wavefields can be decomposed into its frequency components through the discrete Fourier transform for each step in time, followed by summation over all frequencies we were able to recover the snapshot generated in the time domain.

In order to test the frequency domain RTM algorithm, a simpler model was used, shown in Figure 5, where a migrated image was obtained from RTM using the data in the time domain, and in Figure 6 the result of the migration in the frequency domain was obtained through the proposed method. It was also observed that our method using the frequency domain shows better delineated reflectors, especially in the deeper parts of the image, when compared to the conventional RTM image.

The second model is the Marmousi dataset, which is constructed on the basis of a real geological profile, making it a complex geological structure. The dataset generated from this model therefore became a popular choice for advanced migration method tests (Bevc, 1995; Audebert et al., 1997). 


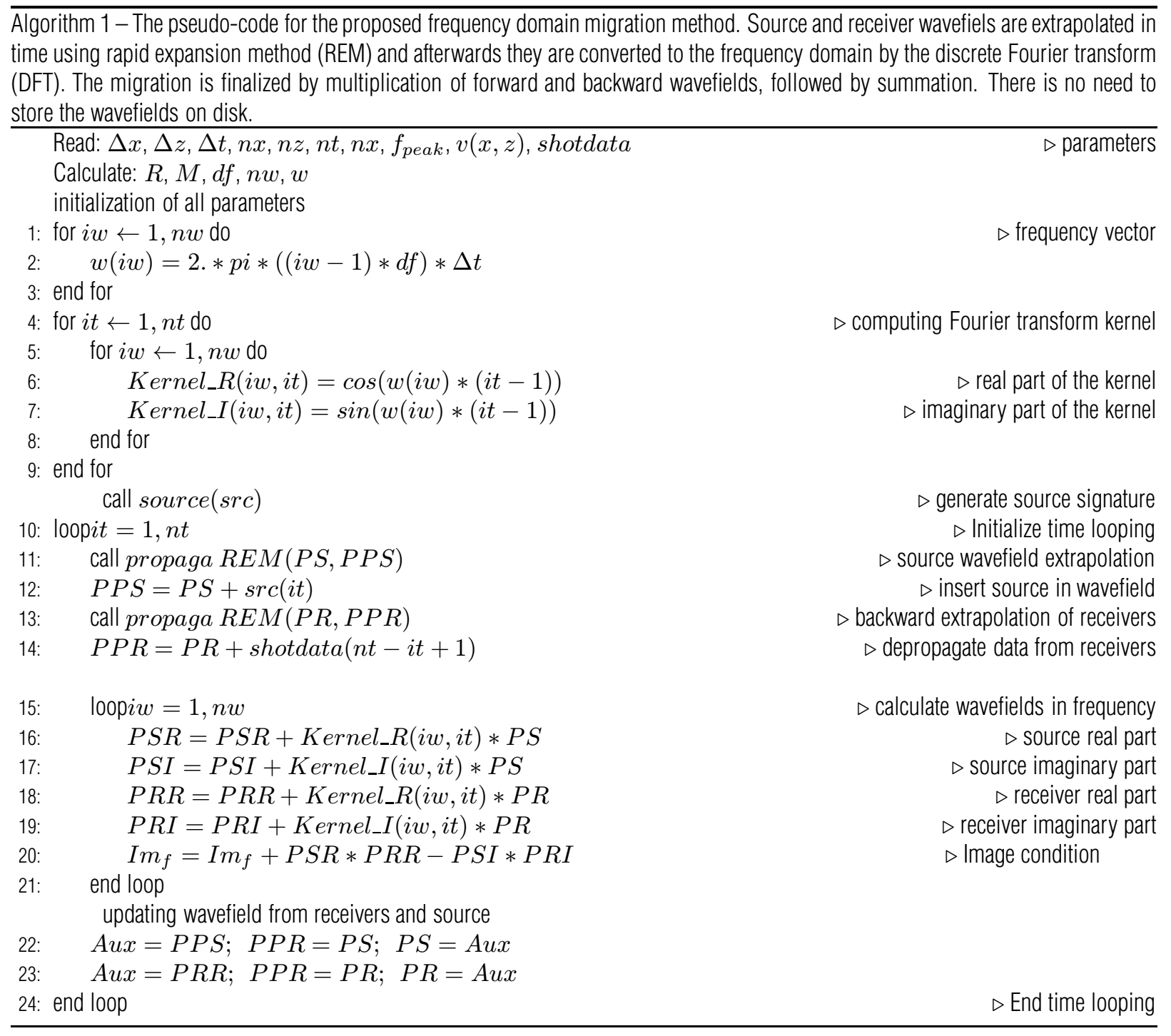

The velocity field of the Marmousi model, showed in Figure 7 , has 369 points in the horizontal direction $(x)$ and 375 in the vertical direction $(z)$ and the spacings are $\Delta x=25 \mathrm{~m}$ and $\Delta z=8 \mathrm{~m}$. The velocities vary from $1500 \mathrm{~m} / \mathrm{s}$ to $5500 \mathrm{~m} / \mathrm{s}$. The Marmousi dataset used here has 240 shots, each shot with 96 traces, each trace with 725 samples where the time sampling interval is $4 \mathrm{~ms}$.

To understand the computational performance of the proposed migration method in frequency domain, the result from conventional RTM will be compared. In conventional RTM, without using the checkpoint technique, it is necessary to record all snapshots from the source wavefield, having to save on disk or in memory a data cube of dimensions $(n x, n z, n t)$. To do the back propagation of the receivers there is another time loop in which the data are injected in a reverse way, and at each step in time, the source data of the direct propagation must be accessed to construct the migrated image, which is obtained through the zero-lag cross-correlation of the source and receiver wavefields.

For the proposed method, it is not necessary to use two loops in time, one for direct propagation and one for reverse propagation. In our implementation, the recorded data is back propagated while the source wavefield is propagated using only one time loop, therefore propagating the source and back propagating the receivers at the same time loop and then transforming both wavefields to the frequency domain.

For each step in time, the wavefields must be stored in four arrays, one for the real part and one for the imaginary part for each complex wavefield, as shown in the pseudo-code (Algorithm 1). 


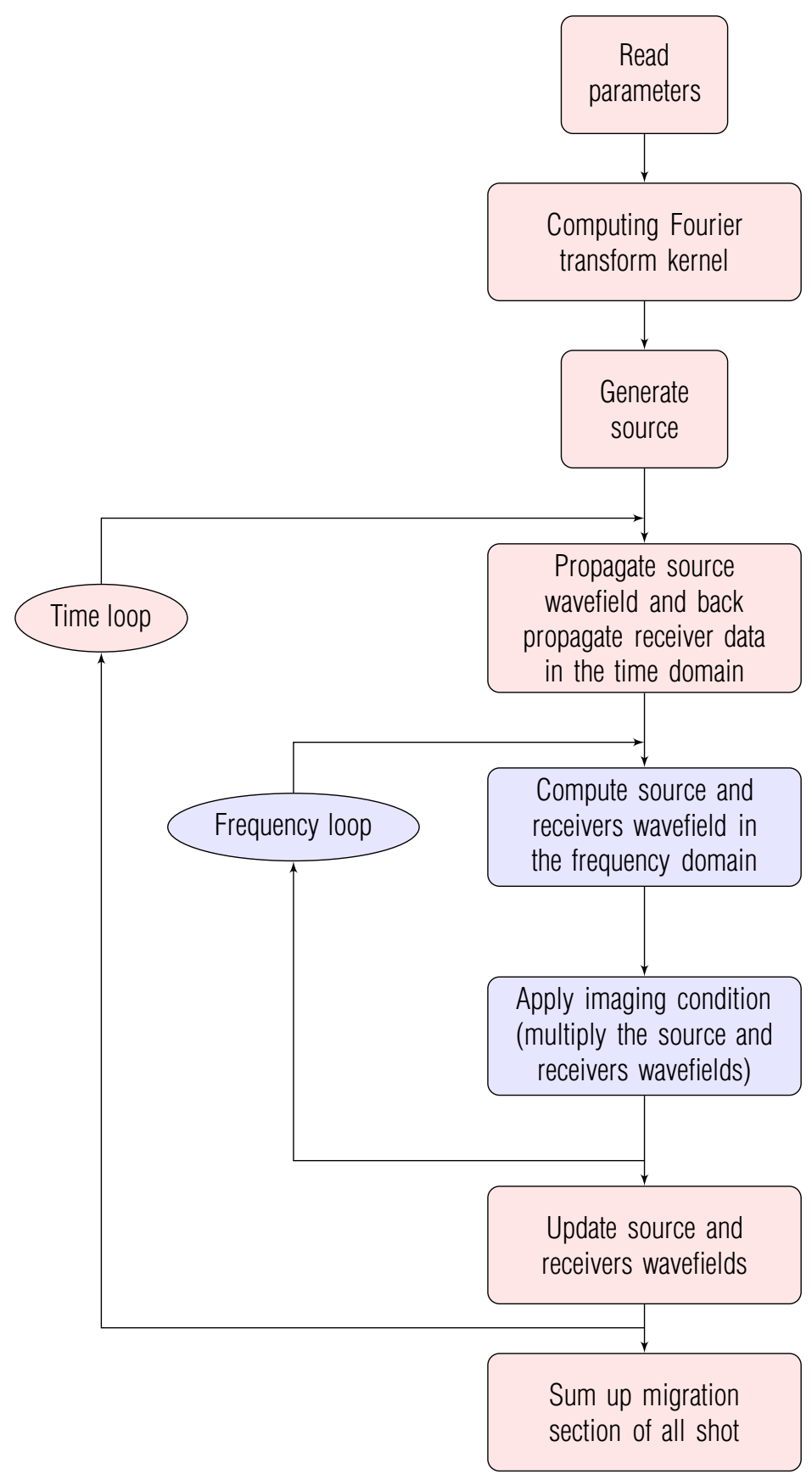

Figure 2 - Flowchart of the proposed frequency domain RTM. For each shot gather forward and backward propagated wavefields are transformed to frequency domain by the discrete Fourier transform. The imaging condition in the frequency domain consists of a simple multiplication of the wavefields at each frequency, followed by summation of all selected frequencies.

Comparing the two methods, taking as an example the Marmousi data, where: $n t=750, d t=0.004 \mathrm{~s}$ and $f_{\text {peak }}=20 \mathrm{~Hz}$, we have $n f=120$, since $n f=f_{\max } / d f$, where $f_{\max }=$ $2 \times f_{\text {peak }}$, and $d f=1 /(n t \times d t)$. Thus, the amount of memory used to save the $(n x, n z, 725)$ cube data of source snapshots is approximately $34 \%$ greater than when compared to the four arrays $(n x, n z, 120)$ used for the real and imaginary parts of the source and receiver wavefields. This represents a substantial improvement in terms of memory occupation when compared to the computational memory the conventional RTM in time requires. 


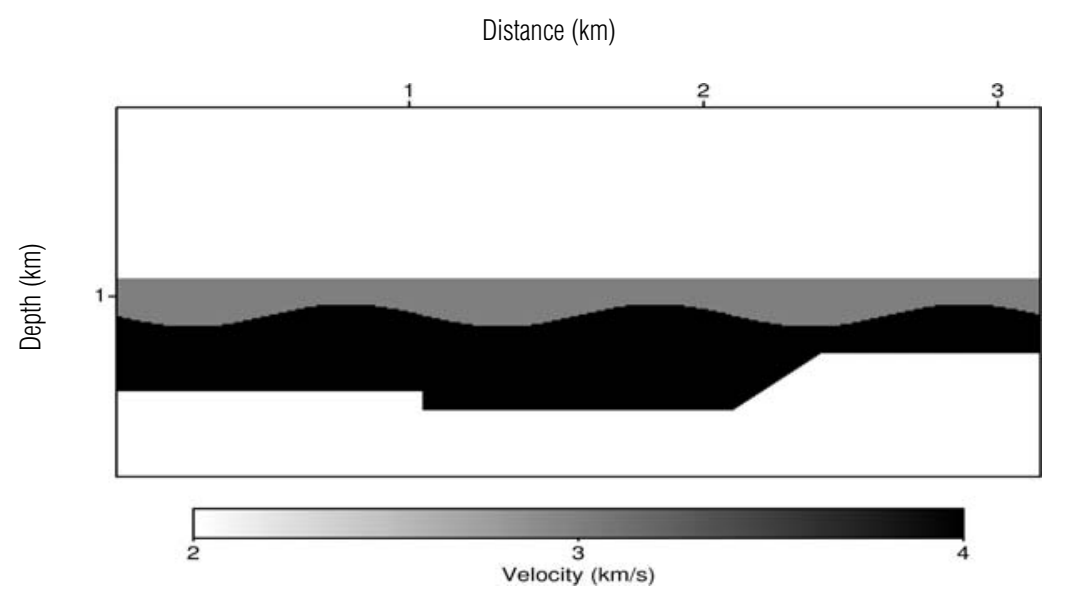

Figure 3 - Depth velocity model used for forward modeling and reverse time - from Tessmer (2011).

For 3D data, RTM typically demands a great amount of memory to store all snapshots of the source wavefield, or when using the checkpoint technique, aside from memory, a large amount of disk space must also be used both to write and read the data.

In the proposed method for 3D data, a large amount of memory will also be required. However, the proposed method uses a significantly reduced amount of memory when compared to time domain RTM methods.

The corresponding pseudo-code is shown in Algorithm 1 and an algorithm description is also presented through the flowchart shown in Figure 2.

To verify the efficiency of the proposed method in time reverse migration, the data presented below were processed in a cluster using 121 nodes under the same hardware configuration.

For the propagation in the time domain we use the checkpoint methodology, where some snapshots of the source wavefield are recorded (saved in disk), and within the back propagation process, propagation of the source wavefield is done for the snapshots that were not saved to disk. Then, a crosscorrelation of the wavefields is done to obtain the migrated image. If there are a large number of checkpoints, these snapshots should be recorded on disk and accessed during migration. Otherwise, they can also be stored in the memory (RAM) of the nodes that will be used.

In order to simulate the RTM migration processing without using the checkpoint methodology, we present the result of Figure 8 where the RTM image is obtained through the conventional method, that is, writing all the wavefields from the source to disk, totaling 725 snapshots, and later accessing them for the construction of the migrated image. For this migration in time the frequency band used was from 0 to $40 \mathrm{~Hz}$ in order to compare with the frequency migration results from the proposed method.

To reduce the overhead of storing snapshots, checkpointing strategy will be used. In this case, the variable N-check indicates the number of wavefield snapshots recorded, to be later accessed, recomputed the missing snapshots by forward time stepping and correlated with the back propagated field to construct the migrated image.

In Figures 9 and 10 we present the results using the checkpoint strategy. Figure 9 with $\mathrm{N}$-check $=290$ source field snapshots, and Figure 10 with $\mathrm{N}$-check=58 source field snapshots. The processing time decreases as the number of snapshots recorded decreases, which can be observed in Table 1. But after 58 recorded fields, the final image begins to lose quality, shown in the result of Figure 11 where only $\mathrm{N}$-check=16 snapshots of the source wavefield were recorded.

Figure 12 shows the RTM result without the use of the checkpoint again, that is, by recording all the source fields in the node RAM, and thus providing the best result in processing time.

The result using our proposed method is shown in Figure 13, where the image is constructed from the frequency domain data. When we look at the best result obtained in the time domain presented in Figure 12, we can see that the data migration in the frequency domain shows an image with more delineated reflectors and a higher resolution than the image obtained with conventional RTM

Using the proposed method allows for migration in a specific frequency band, varying the steps within this same band, like for example, taking larger steps at lower frequencies and shorter steps at higher frequencies, which allows for a smaller matrix to 


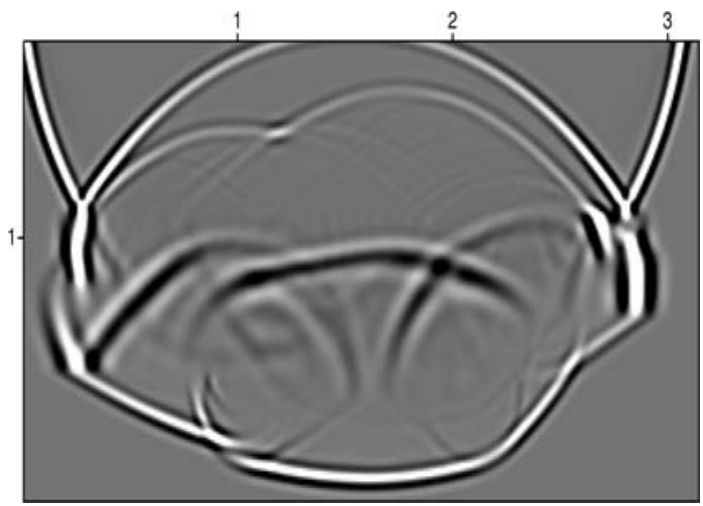

(a)



(c)

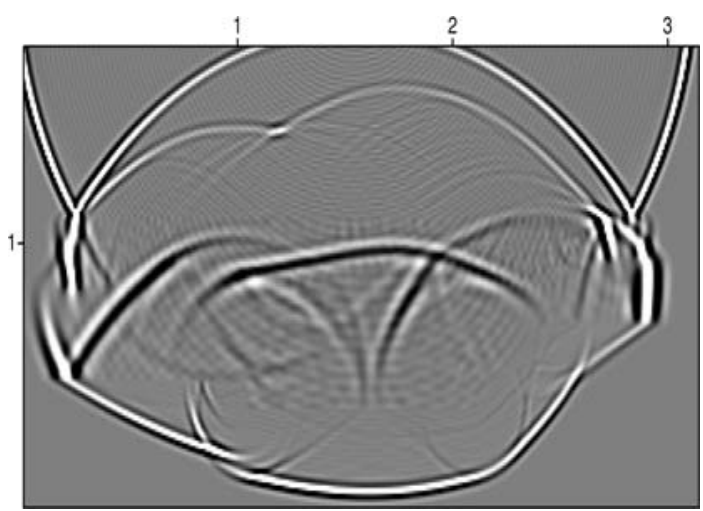

(e)

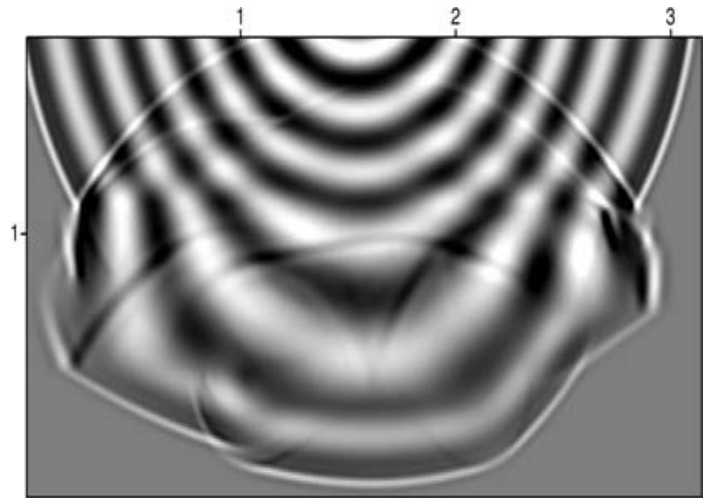

(b)

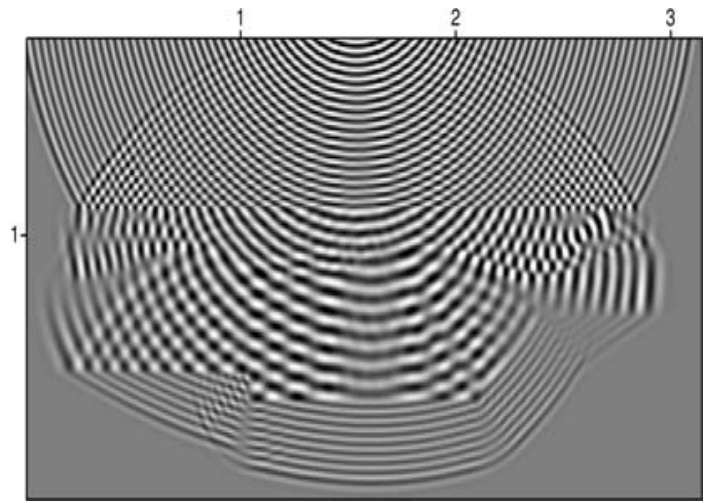

(d)

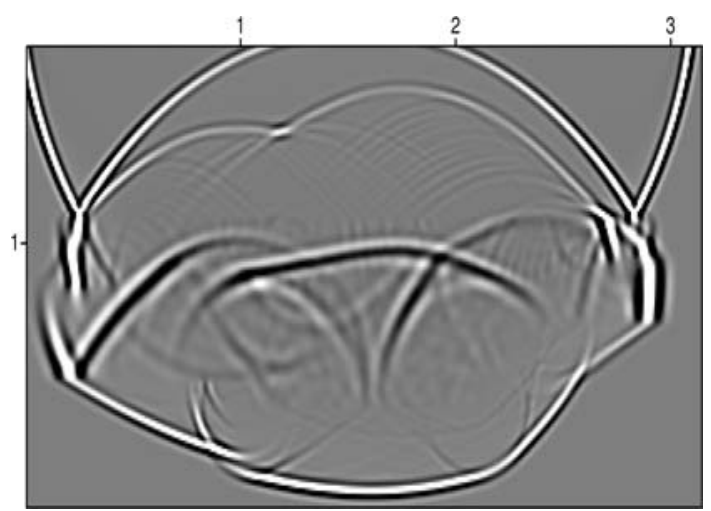

(f)

Figure 4 - Snapshot for the four-layer model at time $0.84 \mathrm{~s}$ shown in the Figure 3. Figure 4(a) in time domain, all other images are wavefield decompositions and frequency sums at intervals (b) 0 to $10 \mathrm{~Hz}$, (c) 0 to $30 \mathrm{~Hz}$, (d) 0 to $60 \mathrm{~Hz}$, (e) 0 to $90 \mathrm{~Hz}$, (f) 0 to $120 \mathrm{~Hz}$.

store the wavefield in the frequency domain. Figure 14 shows the migration result in the frequency range 0 to $40 \mathrm{~Hz}$, with varying steps in the frequency, beginning with longer steps that decrease as the frequency increases, with a greater concentration at the high frequency components. Therefore, we can decrease the migration time processing in the frequency domain by decreasing the num- ber of frequencies, but maintaining the data frequency band.

The time sets spent to obtain the migrated images are presented in Table 2, which shows that when we compare the proposed method to any of the time results with checkpointing, that is, where the source snapshots are written to disk, it showed a better perform in terms of computational processing time. When 




Figure $\mathbf{5}$ - Migration result in the time domain for the model shown in Figure 3.

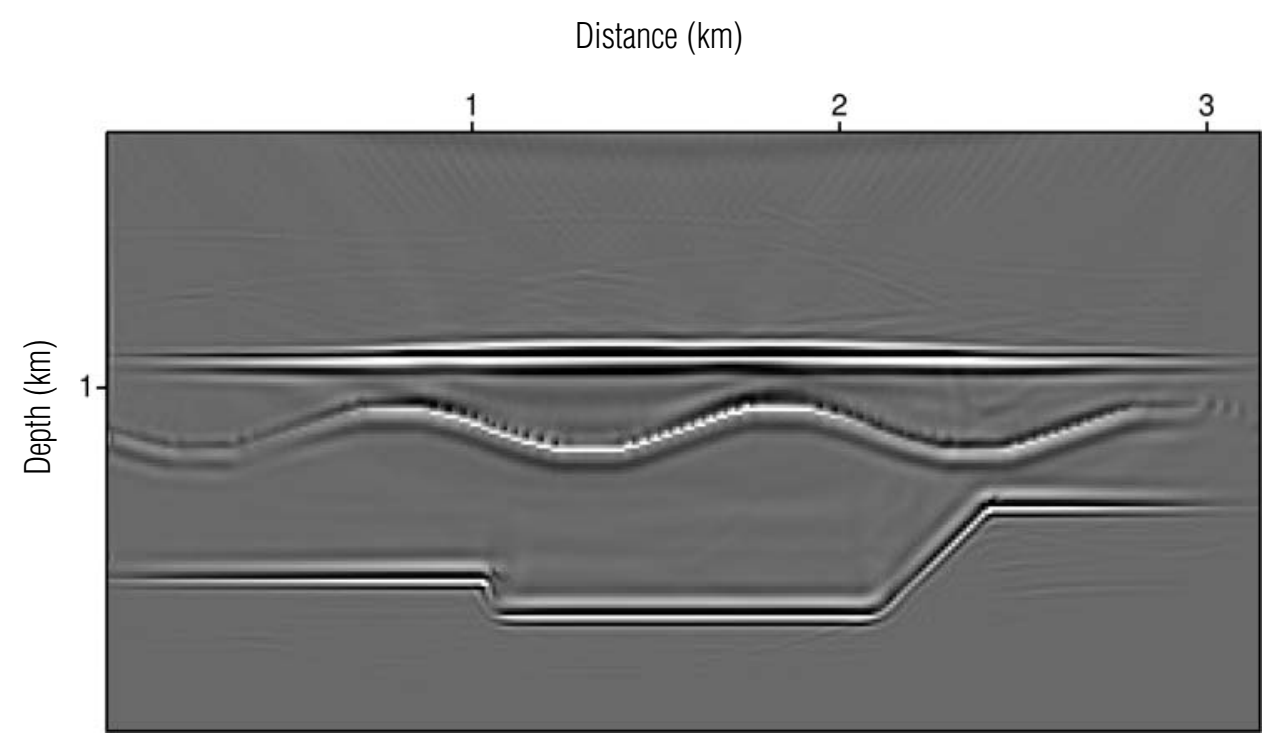

Figure 6 - Migration result in the frequency domain for the model shown in Figure 3.

Table 1 - Processing time of RTM in time domain for Marmousi dataset with a parallel code using a cluster with 121 nodes.

\begin{tabular}{ccc}
\hline N-check & Time (min) & Result in figure \\
\hline 725 & 144 & 8 \\
290 & 48.7 & 9 \\
58 & 20.5 & 10 \\
16 & 20.5 & 11 \\
No checkpoints & 15.2 & 12 \\
\hline
\end{tabular}




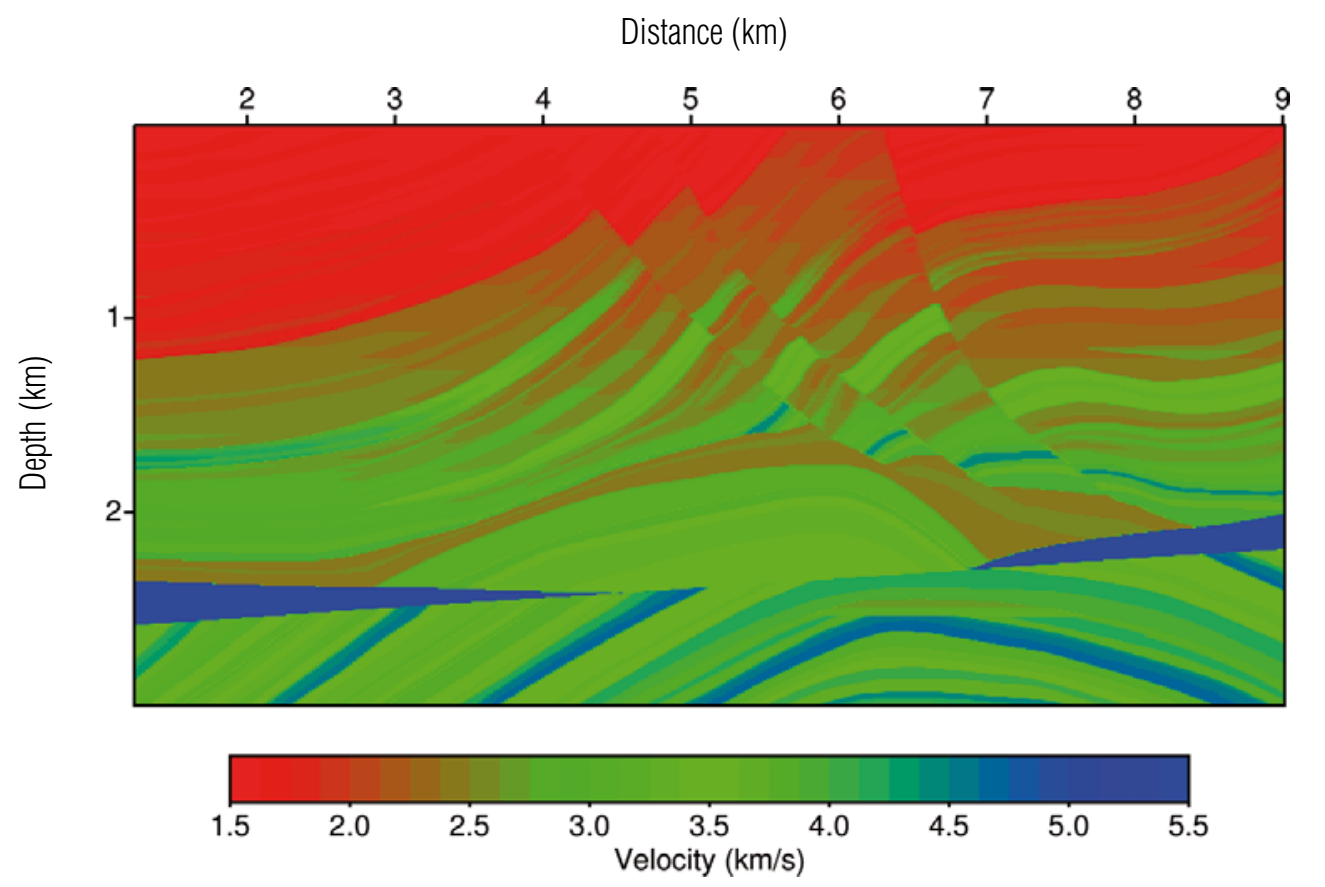

Figure 7 - Subsurface Marmousi velocity model used for forward modeling and reverse time migration.

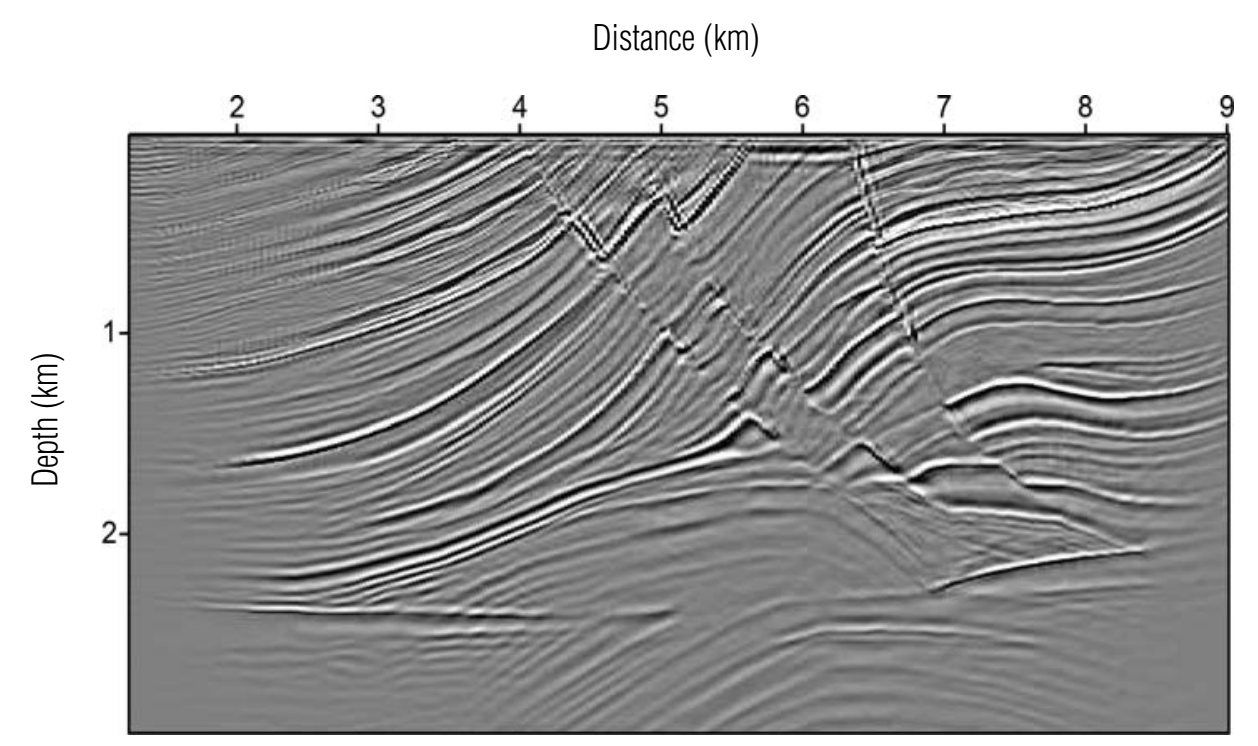

Figure 8 - Reverse time migration using time domain, zero-lag cross-correlation imaging condition and without using checkpoint methodology. All source snapshots were writing to disk and later accessing them for the construction of the migrated image.

we compare the method to the case where source snapshots are saved to memory (RAM), the frequency method presents a little higher result in processing time. However, the frequency domain RTM result presents the reservoir target (in the deeper part of the image) with a greater image contrast in comparison with the migration result in the time domain.
The Marmousi data is relatively small and the time spent writing/reading the data on the disk, that is, when no checkpointing strategy is used, has produced a significant effect on the whole migration method in terms of processing time. In case checkpoints are implemented in the RTM in time domain the computational time decrease but we still need to used a reasonable 


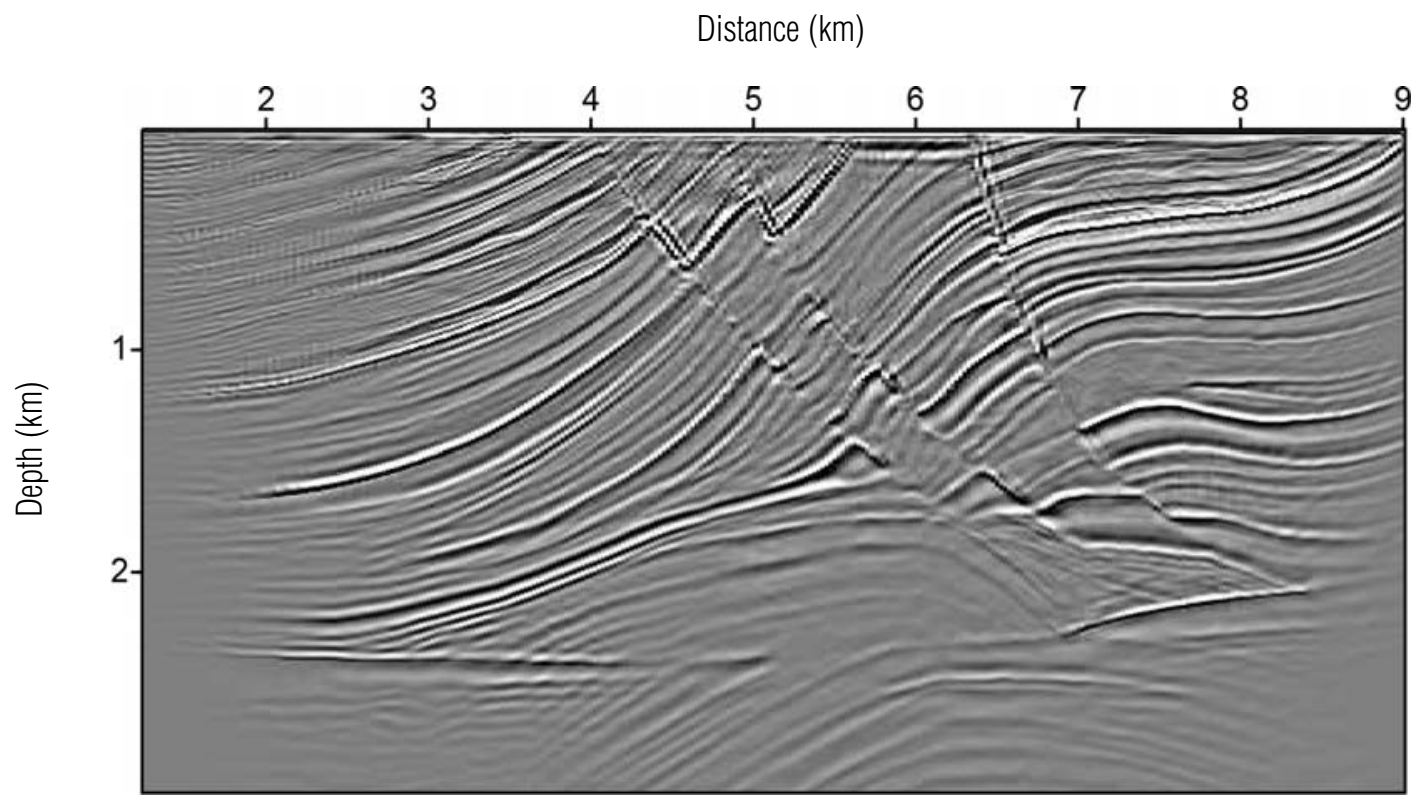

Figure 9 - Migration in time domain for Marmousi using checkpointing strategy where N-check=290 snapshots were saved in disk. During the reverse time computations and correlation, the missing snapshots are recomputed by forward time stepping from stored snapshots.

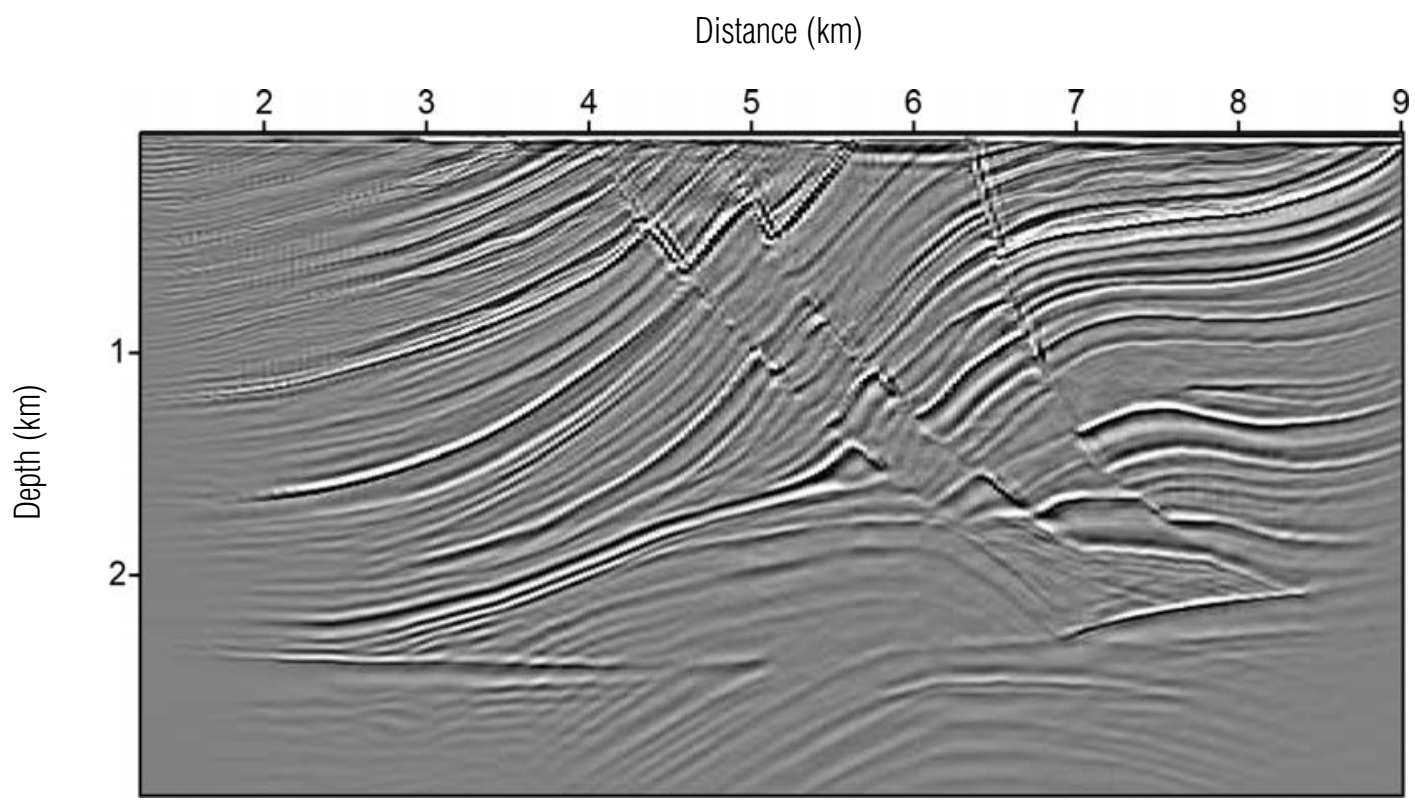

Figure 10 - Migration in time domain for Marmousi, with checkpointing strategy where N-check=58 snapshots saved to disk. The missing snapshots are recomputed during the reverse time computations and correlation by forward time stepping from stored snapshots.

number of checkpoints to guarantee the quality of the final migration image. Differently, our proposed method of migration in the frequency domain requires less computational memory and no checkpoints. From Figure 2 we can notice that it is more efficient when compared with the RTM in time domain implemented here.

The proposed method can be applied using varying step lengths in the frequency within of migration band, making this 


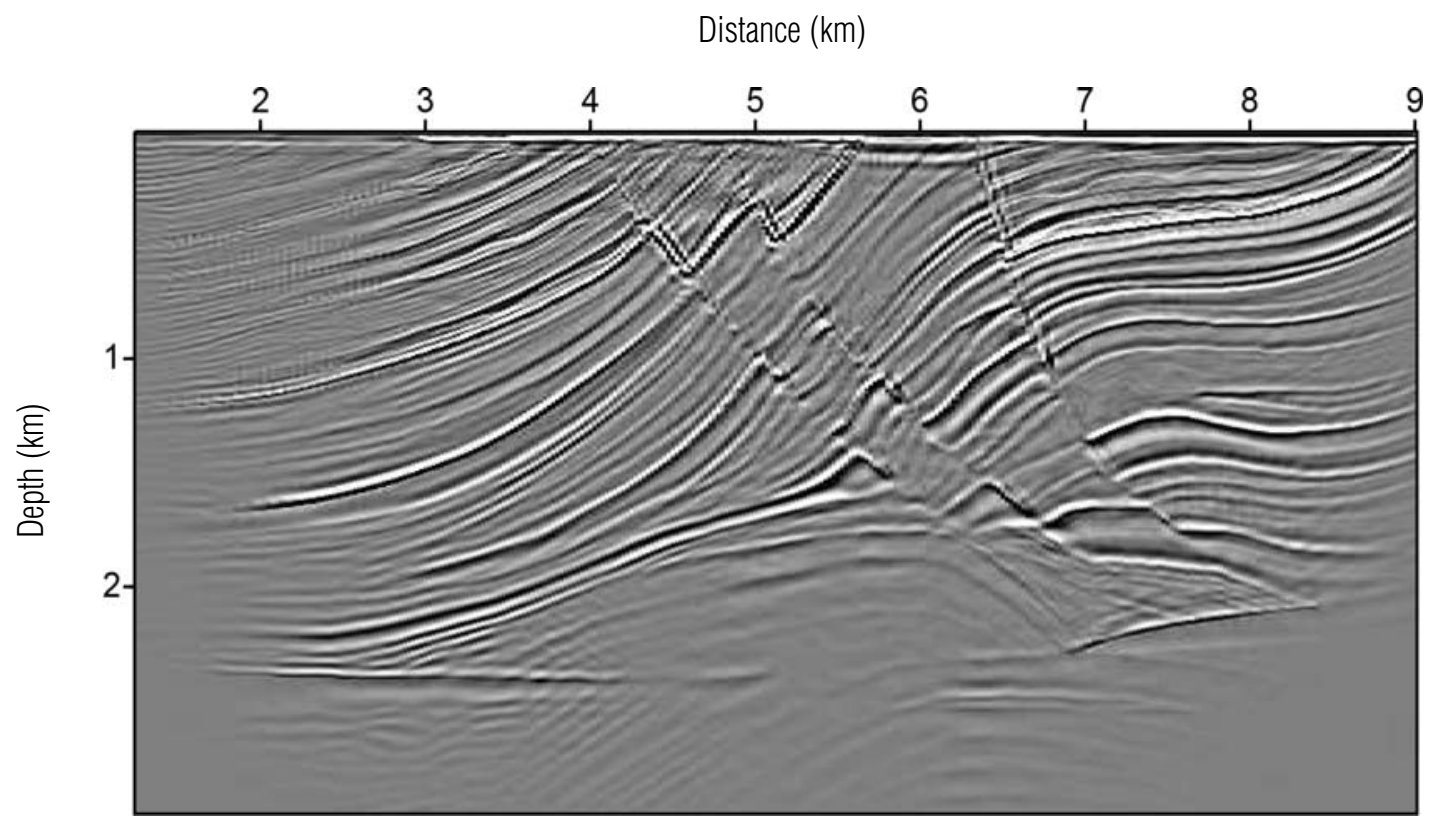

Figure 11 - Migration in time domain for Marmousi using checkpointing strategy where only N-check=16 snapshots were saved in disk. During the reverse time computations and correlation, the missing snapshots are recomputed by forward time stepping from stored snapshots.

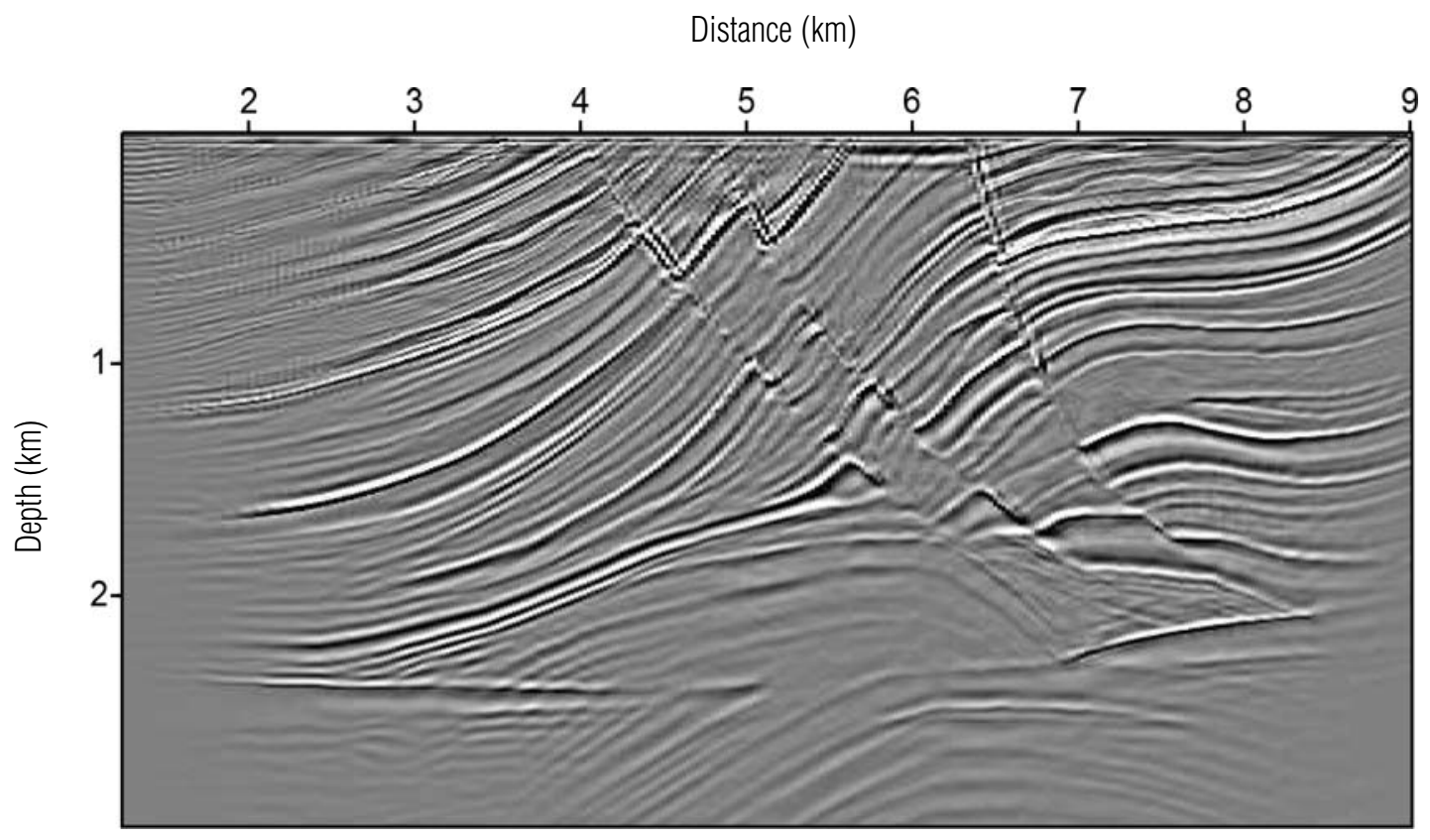

Figure 12 - Migration in time domain for Marmousi without the use of checkpointing saving all source snapshots in the memory (RAM) of each compute node.

a favorable method for the application of the complete wavefield inversion (FWI) process, where the gradient calculation is equivalent to an RTM migration, permitting gradient optimiza- tion and making the process of updating the model parameters an efficient process, specially when applied for a large volume of seismic data. 




Figure 13 - Migration in frequency domain for Marmousi using the proposed method with a band of frequency from 0 to $40 \mathrm{~Hz}$ with a number of frequency sampled equal to 118 .

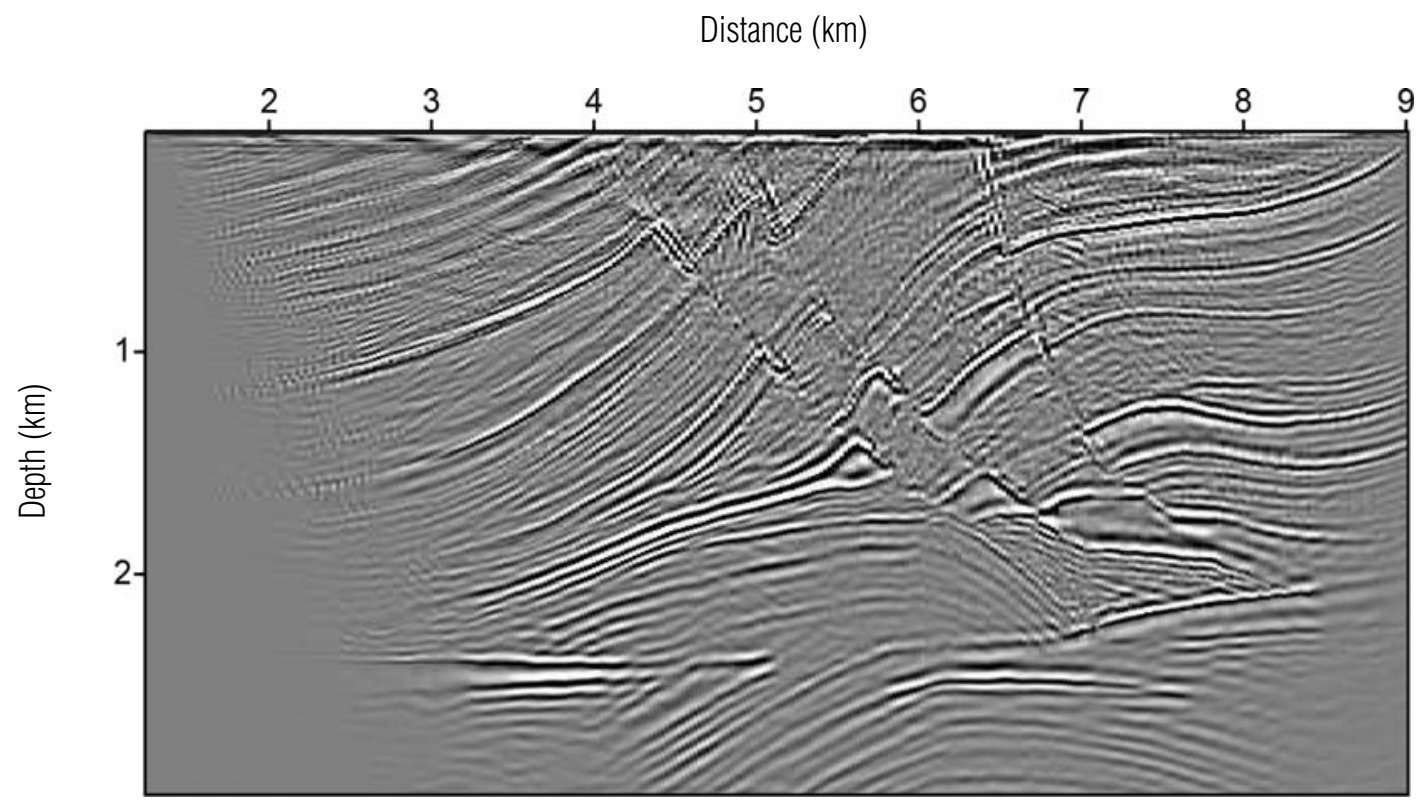

Figure 14 - Migration in frequency domain for Marmousi in the range of 0 to $40 \mathrm{~Hz}$ band, with varying steps in the frequency, beginning with longer steps that decrease as the frequency increases, with a greater concentration at the high frequency components. In this case was migrated 55 frequency components.

\section{Real Data Result}

The Gulf of Mexico is one of the regions with the highest oil and gas productivity worldwide and the presence of salt structures in this region is related to the trapping of hydrocarbons in several ways and must be taken into account for the correct interpretation of reservoirs (Chowdhury et al., 2007). Here we use real 2D-data from the Gulf of Mexico, obtained along a marine canyon of the Mississippi delta located south of Louisiana (USA) in the central gulf area. The diek features a central salt pad with horizontal rock 
Table 2 - Processing time of RTM in frequency domain for Marmousi dataset with a parallel code using a cluster with 121 nodes.

\begin{tabular}{cccc}
\hline Frequency band $(\mathrm{Hz})$ & Number of frequencies & Time $(\mathrm{min})$ & Result in figure \\
\hline $0-40$ & 118 & 18.5 & 13 \\
$0-40$ & 55 & 16.5 & 14 \\
\hline
\end{tabular}



Figure 15 - Gulf of Mexico velocity model.

layers above and below as shown in the Gulf of Mexico speed field (Fig. 15). For this figure, the horizontal grid has 1185 points in the horizontal direction $(x)$, while the vertical grid has 1000 in the vertical direction $(z)$, spacings is $\Delta x=26.6 \mathrm{~m}$ and $\Delta z=$ $6.7 \mathrm{~m}$, respectively. In this model, velocity range from $1500 \mathrm{~m} / \mathrm{s}$ to $5500 \mathrm{~m} / \mathrm{s}$. The data has 1000 shots, with 180 traces, each trace with 1501 samples of time.

The presence of a salt structure make it hard to image deep layers, since it is a high-velocity barrier, which prevents waves scattered by deep reflectors from reaching the surface receivers. This is the ideal situation to test the proposed frequency domain migration algorithm.

The result of the migration in the time domain is shown in Figure 16, as well as the result of the migration in the frequency domain Figure 17. Both results well image the salt cushion. This result for the proposed method is very significant, since the complexity of processing a real data is much greater when compared to a synthetic data, because in the actual marine data we have the presence of coherent noises due to the infrastructure of the ship itself and the presence from others, from random noise from the waves and from the tow of acquisition cables, among others, which are not present in the synthetic data.

The signature of the seismic source used is another factor that can influence the quality of the migration of the seismic data, since in the modeling we use a pulse of the type Ricker different from the pulse of the seismic source if the difference between the pulse used in the modeling and the generated pulse because the seismic source is large, the migration result will change. In this way, our result with real data quantifies the proposed algorithm as a suitable method for the processing of real data.

\section{CONCLUSIONS}

We proposed an alternative procedure to generate seismic migration data in the frequency domain using rapid expansion method to extrapolate wavefields. During extrapolation, the source and receiver wavefields are transformed into the frequency domain through the efficient implementation of the discrete Fourier trans- 


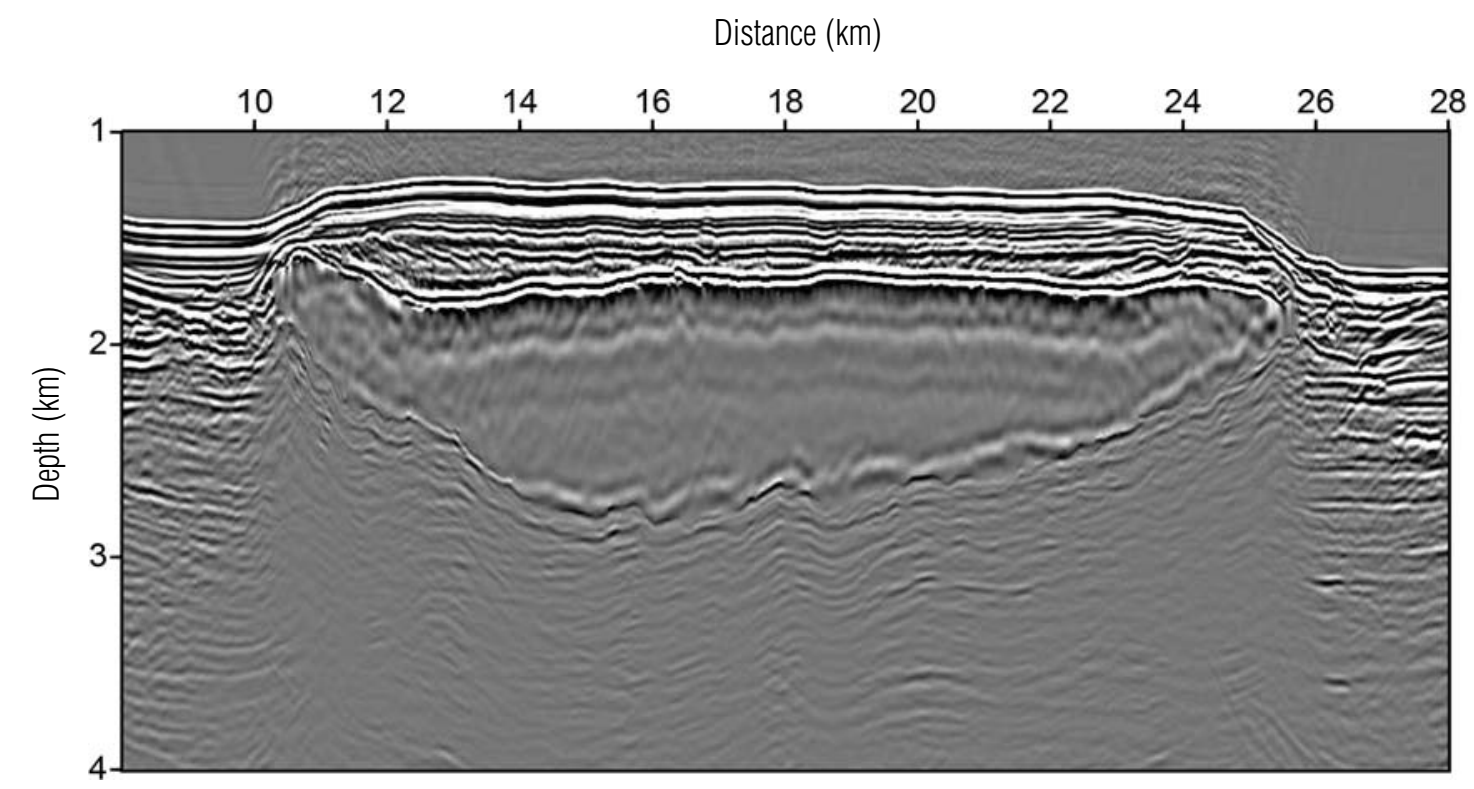

Figure 16 - Migration result in time domain for Gulf of Mexico, with the processing time of $8 \mathrm{~h}$ and $24 \mathrm{~min}, i c h e c k=4, n c h e c k=752, f_{\max }=27 \mathrm{~Hz}$, processed in 101 nodes.

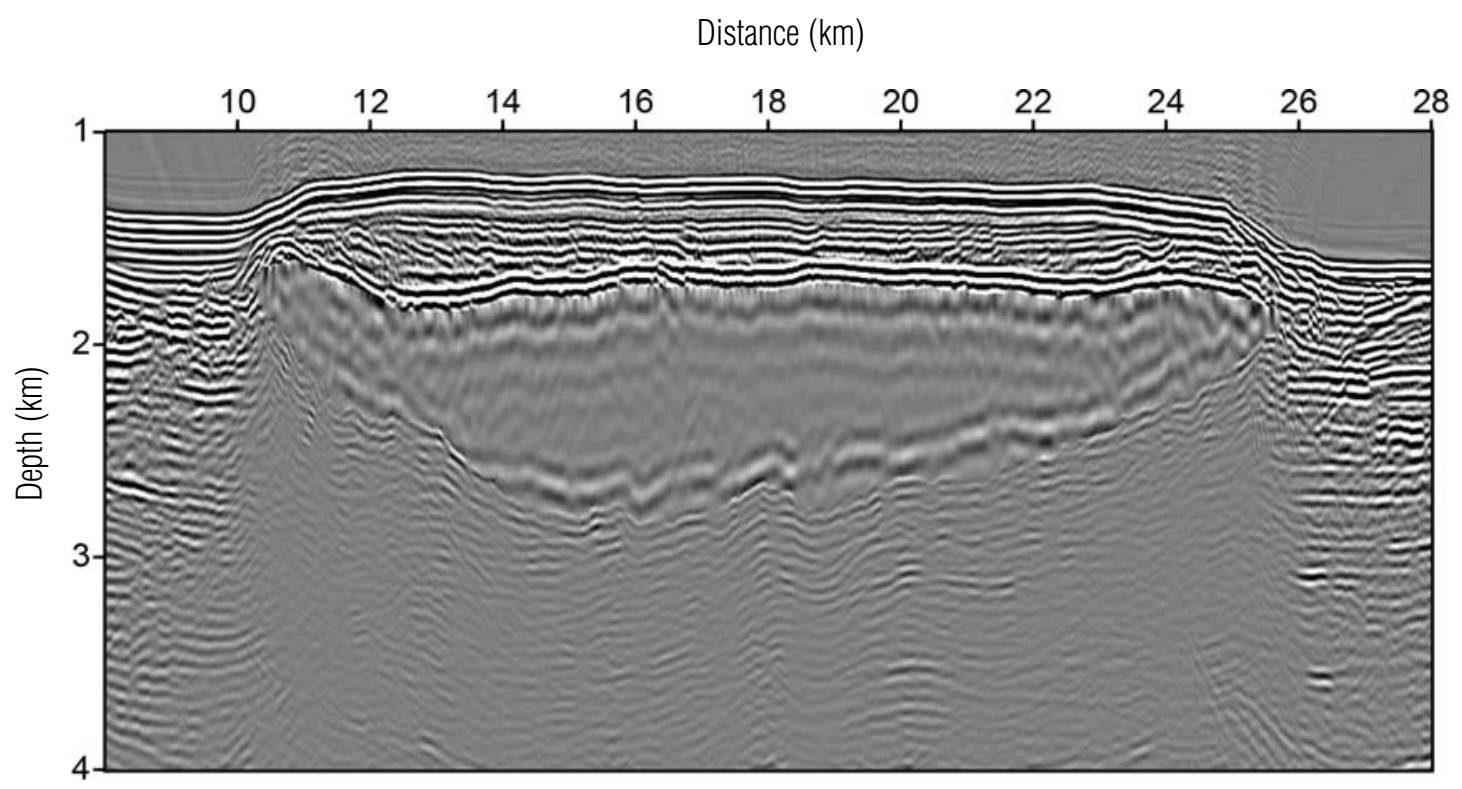

Figure 17 - Migration result in frequency domain for Gulf of Mexico, with the processing time of $9 \mathrm{~h}$ and $9 \mathrm{~min}, n w=164, f_{\max }=27 \mathrm{~Hz}$.

form. In this way, the migrated image is obtained simply by multiplying of the extrapolated fields for each frequency component.

In our method, the extrapolation of source and backward propagation of receivers are performed in a single time loop, transform to the frequency domain and thus producing a significant reduction of memory requiments and removing the need to write/read the source wavefield on disk. Furthermore, the pro- posed method does not need to use the checkpoint strategy for the source snaspshots and becomes a more effcient method in comparison with the standard RTM.

The alternative migration procedure, based on a time-tofrequency domain transformation, applied in this work can produce accurate results with a high quality, especially for complex geologies, as shown in our results, which considers the real data 
of the Gulf of Mexico. Moreover, our tests with the Marmoussi dataset showed that our method is more computationally efficient when compared to conventional RTM method. The results obtained it can be concluded that the computational efficiency of the proposed method in relation to the conventional method using the checkpoint would be better for the 3D case.

The proposed method shown in this work, that can be used to construct Green's functions optimally, where it is not necessary to calculate the Green function of the source with each receiver separately as the standard, and the function of Green from the source with all the receivers at one time, making its application wide for the various forms of operators of migration in the frequency domain.

\section{ACKNOWLEDGEMENTS}

This research was supported by CNPq, CAPES and INCTGP/CNPq. The facility support from CPGG/UFBA is also acknowledged.

\section{REFERENCES}

AUDEBERT F, NICHOLS D, REKDAL T, BIONDI B, LUMLEY DE \& URDANETA H. 1997. Imaging complex geologic structure with single-arrival kirchhoff prestack depth migration. Geophysics, 62(5): 1533-1543.

BAYSAL E, KOSLOFF DD \& SHERWOOD JWC. 1983. Reverse time migration. Geophysics, 48: 1514-1524.

BEVC D. 1995. Imaging Under Rugged Topography and Complex Velocity Structure. Ph.D. Thesis, Stanford University. $97 \mathrm{pp}$.

CHOWDHURY A, BORTON L \& NOPEC T. 2007. Salt geology and new plays in deep-water Gulf of Mexico. In: 2007 AAPG Annual Convention and Exhibition.

CHU C\& STOFFA P. 2012. Efficient 3D frequency response modeling with spectral accuracy by the rapid expansion method. Geophysics, 77(4): T117-T123. doi: 10.1190/GE02011-0415.1.

CLAERBOUT JF. 1971. Toward a unified theory of reflector mapping. Geophysics, 36(3): 467-481.

DAI W, FOWLER P \& SCHUSTER GT. 2012. Multi-source least-squares reverse time migration. Geophysical Prospecting, 60(4): 681-695.

FURSE CM. 2000. Faster then Fourier: Ultra-efficient time-to-frequencydomain conversions for FDTD simulations. IEEE Antennas and Propagation Magazine, 42: 24-34. doi: 10.1109/74.894179.

HAMMAD HI. 2010. Waveform inversion for areas with complex near surface. Msc. Thesis, University of Calgary, Calgary, Alberta. 102 pp.

HEMON C. 1978. Equations d'onde et modeles. Geophysics Prospecting, 26: 790-821.
KOSLOFFD, FILHO AQ, TESSMERE \& BEHLEA. 1989. Numerical solution of the acoustic and elastic wave equations by a new rapid expansion method. Geophysical Prospecting, 37: 383-394.

LOEWENTHAL D \& MUFTI RI. 1983. Reversed time migration in spatial frequency domain. Geophysics, 48: 627-635.

MAAREF N, MILLOT P, FERRIÈRES X, PICHOT C \& PICON 0. 2008. Electromagnetic imaging method based on time reversal processing applied to through-the-wall target localization. Progress In Electromagnetics Research M, 1: 59-67.

McMECHAN GA. 1983. Migration by extrapolation of time-dependent boundary values. Geophysics Prospecting, 31: 413-420.

NIHEI KT \& LI X. 2007. Frequency response modelling of seismic waves using finite difference time domain with phase sensitive detection (TD-PSD). Geophysical Journal International, 169(3): 1069-1078. doi: 10.1111/gji.2007.169.issue-3.

OPERTO S, VIRIEUX J, AMESTOY P, L'EXCELLENT JY, GIRAUD L \& ALI HBH. 2007. 3D finite-difference frequency-domain modeling of viscoacoustic wave propagation using a massively parallel direct solver: A feasibility study. Geophysics, 72(5): SM195-SM211.

PAN J. 2006. A migration approach based on summation of individual trace contributions and wavefield extrapolation. In: SEG Technical Program Expanded Abstracts 2006, pp. 2421-2425. doi: 10.1190/1.2370021.

PESTANA R \& STOFFA P. 2009. Rapid expansion method (REM) for time stepping reverse time migration (RTM). In: 79th Annual Inter. Meeting, SEG, Expanded Abstracts, pp. 2819-2823.

PESTANA RC \& STOFFA PL. 2010. Time evolution of wave equation using rapid expansion method. Geophysics, 75: T121-T131.

REN H, WANG H \& CHEN S. 2013. Least-squares reverse time migration in frequency domain using the adjoint-state method. Journal of Geophysics and Engineering, 10(3): 035002.

SHABELANSKY AH. 2007. Full wave inversion. Ph.D. Thesis, Tel-Aviv University, Israel. 58 pp.

SIRGUE L, ETGEN J \& ALBERTIN U. 2008. 3D frequency domain waveform inversion using time domain finite difference methods. In: 70th EAGE Conference and Exhibition incorporating SPE EUROPEC 2008.

SONG J. 2008. The optimized expression of a high dimensional function/manifold in a lower dimensional [in Chinese]. Chinese Scientific Bulletin, 46: 977-984.

SOUBARAS R \& ZHANG Y. 2008. Two-step explicit marching method for reverse time migration. In: 78th Annual Inter. Meeting, SEG, Expanded Abstracts, pp. 2272-2276.

TAL-EZER H, KOSLOFF D \& KOREN Z. 1987. An accurate scheme for seismic forward modelling. Geophysical Prospecting, 35(5): 479-490. doi: 10.1111/j.1365-2478.1987.tb00830.x. 
TESSMER E. 2011. Using the rapid expansion method for accurate timestepping in modeling and reverse time migration. Geophysics, 76: S177S185.

WHITMORE DW. 1983. Iterative depth migration by backward time propagation. In: 53rd Annual Inter. Meeting, SEG, Expanded Abstracts, pp. 382-385.

ZHANG Y \& ZHANG G. 2009. One-step extrapolation method for reverse time migration. Geophysics, 74(4): A29-A33.

Recebido em 6 outubro, 2017 / Aceito em 20 fevereiro, 2018

Received on October 6, 2017 / Accepted on February 20, 2018 